\title{
CONVERGENCE OF DIFFERENCE SCHEMES WITH HIGH RESOLUTION FOR CONSERVATION LAWS
}

\author{
GUI-QIANG CHEN AND JIAN-GUO LIU
}

\begin{abstract}
We are concerned with the convergence of Lax-Wendroff type schemes with high resolution to the entropy solutions for conservation laws. These schemes include the original Lax-Wendroff scheme proposed by Lax and Wendroff in 1960 and its two step versions-the Richtmyer scheme and the MacCormack scheme. For the convex scalar conservation laws with algebraic growth flux functions, we prove the convergence of these schemes to the weak solutions satisfying appropriate entropy inequalities. The proof is based on detailed $L^{p}$ estimates of the approximate solutions, $H^{-1}$ compactness estimates of the corresponding entropy dissipation measures, and some compensated compactness frameworks. Then these techniques are generalized to study the convergence problem for the nonconvex scalar case and the hyperbolic systems of conservation laws.
\end{abstract}

\section{INTRODUCTION}

We are interested in the convergence of finite difference schemes with high resolution for conservation laws

$$
\partial_{t} u+\partial_{x} f(u)=0, \quad u(x, 0)=u_{0}(x) \in L^{2}(\mathbb{R}) \cap L^{\infty}(\mathbb{R}) .
$$

One of the most fundamental and important second-order finite difference schemes is the Lax-Wendroff scheme [11]. It is defined by

$$
\begin{aligned}
u_{j}^{n+1}= & u_{j}^{n}-\frac{\lambda_{n}}{2} \Delta_{0} f\left(u_{j}^{n}\right)+\frac{\lambda_{n}^{2}}{2} \Delta_{-}\left(f^{\prime}\left(\frac{u_{j}^{n}+u_{j+1}^{n}}{2}\right) \Delta_{+} f\left(u_{j}^{n}\right)\right) \\
& +\lambda_{n} \Delta_{-}\left(\beta_{j+1 / 2}^{n}\left|\Delta_{+} f^{\prime}\left(u_{j}^{n}\right)\right| \Delta_{+} u_{j}^{n}\right),
\end{aligned}
$$

where $u_{j}^{n}=u\left(x_{j}, t_{n}\right), t_{n}=\sum_{k=1}^{n} \tau_{k}, x_{j}=j h, \lambda_{n}=\tau_{n} / h, \Delta_{+} u_{j}=u_{j+1}-u_{j}$, $\Delta_{0} u_{j}=u_{j+1}-u_{j-1}, \Delta_{-} u_{j}=u_{j}-u_{j-1}$, and $\beta_{j+1 / 2}^{n}, 0 \leq \beta_{0} \leq \beta_{j+1 / 2}^{n} \leq \beta_{1}<+\infty$, is a smooth function of $u_{j}^{n}$ and $u_{j+1}^{n}$ (e.g. $\beta_{j+1 / 2}^{n}=$ const. in [11]). Moreover, this scheme has to satisfy the Courant-Friedrichs-Lewy condition

$$
\varepsilon_{0}=\max _{n}\left(\lambda_{n} \max _{j}\left|f^{\prime}\left(u_{j}^{n}\right)\right|\right) \leq 1
$$

for stabilization in general.

This scheme is designed to have the following desirable computational features: conservation form, three-point dependence, second-order accuracy on the smooth

Received by the editor April 1, 1996.

1991 Mathematics Subject Classification. Primary 65M12; Secondary 35L65.

Key words and phrases. Conservation laws, convergence, entropy solution, Lax-Wendroff scheme. 
regions of solutions, and stabilization for the convex case for $\beta_{0}>0$ and $\varepsilon_{0} \leq 1$. When $\beta_{j+1 / 2}^{n}=0$, the Lax-Wendroff scheme serves as not only a simple mode for the physical phenomenon of the dissipation-dispersion couplings, but also an example of dispersive schemes that do not converge in the sense of strong topology (cf. [10]). Indeed, as observed by Harten-Hyman-Lax [7] and Majda-Osher [14], [15], the second-order numerical viscosity $\beta_{j+1 / 2}^{n} \geq \beta_{0}>0$ in this scheme is essential to guarantee that the numerical solutions are nonlinearly stable and converge to the physical solutions. The main role of the factor $\left|\Delta_{+} f^{\prime}\left(u_{j}^{n}\right)\right|$ in the third term of the scheme (1.3) is to reduce effectively viscosity in the smooth regions of solutions to produce sharp discontinuities in numerical computations. Such a scheme does not have a TVD property.

The main objective of this paper is to prove the convergence of the Lax-Wendroff approximate solutions to the entropy solutions and to provide an analytical approach for such a convergence analysis for high-order finite difference schemes, which do not preserve BV (and even $L^{\infty}$ bound). This is motivated by the fact that any high-resolution difference scheme cannot, in general, preserve $B V$ for the hyperbolic systems of conservation laws, especially for the nonstrictly hyperbolic case and the multidimensional case. Since our analysis is qualitative, we will always assume that $\beta_{0}$ is suitably large and $\epsilon_{0}$ is suitably small to make our analysis more convenient without loss of our purpose. One can follow our analysis to get the optimal constants for $\beta_{0}$ and $\epsilon_{0}$ from this approach for some concrete equations. Our analysis is based on careful $L^{p}$ estimates of the approximate solutions, $H^{-1}$ compactness estimates of the corresponding entropy dissipation measures, and some compensated compactness frameworks.

In Section 2 we describe some compensated compactness theorems for conservation laws, which guarantee the convergence of the finite difference schemes provided that the corresponding approximate solutions satisfy these frameworks. Since these compactness frameworks do not need the $B V$ estimates, this enables us to carry through our analysis by using the $L^{p}$ estimates and the $H^{-1}$ compactness estimates, which are weaker than the $B V$ estimates in general.

Section 3 is devoted to the estimates of the Lax-Wendroff approximate solutions to the convex scalar conservation laws. We obtain the uniform $L^{p}$ estimates of the approximate solutions and the $H^{-1}$ compactness estimates of the corresponding entropy dissipation measures by analyzing carefully the properties of this scheme and by developing some useful estimate techniques. A global entropy error estimate is also obtained to ensure the consistency of this scheme with the scalar conservation laws.

In our analysis we need a technical assumption of the algebraic growth of flux functions. This is because we do not require the $L^{\infty}$ bound or the BV bound for the Lax-Wendroff approximate solutions to achieve our goal. One difficulty in the analysis is the fact that the dissipation is third-order in the Lax-Wendroff scheme, comparing with the second-order dissipation in the first-order schemes. All these terms need to be carefully combined into a third-order term or a term consisting of square products of second-order differences with a favorable sign. Moreover, the factor in each combined third-order difference needs to be bounded by the growth factor in the dissipative term in each cell. Some special treatment is also made since the Lax-Wendroff scheme is a three-point scheme. A requirement of small CFL number is made here to use the grid ratio $\lambda$ to control some growth factors. 
In order to get the compactness of entropy dissipation measures, we estimate certain entropy inequalities with a higher growth rate.

These techniques are generalized to study the convergence of the Lax-Wendroff scheme for the nonconvex scalar case in Section 4 and for the hyperbolic systems of conservation laws in Section 5, and to prove the convergence of the Richtmyer scheme [22] and the MacCormack scheme [13]-two step versions of the Lax-Wendroff schemes in Section 6, which are widely used in industry and engineering.

In connection with earlier work on the Lax-Wendroff type schemes, we recall that Majda and Osher [14], [15] showed the $L^{2}$-stability for general scalar conservation laws, the entropy consistency for the boundedly convergent approximate solutions for the semi-discrete cases as well as the complete-discrete scheme for the timeindependent cases of general systems endowed with a convex entropy, the efficient choices of artificial viscosity such as the switching techniques, and the validity of the CFL number in their analysis. Many of their techniques have been melted into our analysis. We also refer to [25] for the stability of the local discrete shock profile for the Lax-Wendroff scheme.

Regarding work on the convergence analysis of full discrete high-resolution finite difference schemes, we refer to [4] and the references cited therein for the flux-limit schemes with slope modification or antidiffusive flux approach, which preserve $L^{\infty}$ bound. Since the Lax-Wendroff type schemes do not have a TVD property, our analysis consists of two steps: One is to prove the convergence, which is an essential difficulty here and is, however, automatically ensured by the Helly principle for the TVD or TVB schemes, and the other is to verify the entropy consistency. The convergence analysis of TVD or TVB schemes focus mainly upon the second step for the scalar case, that is, the consistency proof. The convergence of a class of semi-discrete generalized MUSCL schemes for the strictly convex case was obtained in [19]. For the semi-discrete MUSCL scheme for the convex scalar conservation laws, some consistency results were announced in [12], [31].

\section{COMPaCtNess Frameworks}

In this section we discuss some compactness frameworks for the approximate solutions for subsequent developments. A pair of functions $(\eta(u), q(u))$ is called an entropy-entropy flux pair if they satisfy $q^{\prime}(u)=\eta^{\prime}(u) f^{\prime}(u)$. For the scalar case, any function is an entropy function. In the following theorem and the analysis in Section 3 and Section 4 , we will denote entropy $\eta_{0}(u)=\frac{1}{2} u^{2}$ for the convex case and $\eta_{0}(u)=f(u)$ for the general case.

Theorem 2.1. Consider the scalar conservation laws (1.1) satisfying meas $\{u$ : $\left.f^{\prime \prime}(u)=0\right\}=0$. Let $u_{h}(x, t)$ be numerical approximate solutions of (1.1) satisfying the following conditions:

(1) $u_{h}$ is bounded in $L^{p}$ for some $p \geq 2$ and $\left(f\left(u_{h}\right), \eta_{0}\left(u_{h}\right), q_{0}\left(u_{h}\right)\right)$ is bounded in $L_{l o c}^{2}$

(2) The dissipation measures $\partial_{t} u_{h}+\partial_{x} f\left(u_{h}\right)$ and $\partial_{t} \eta_{0}\left(u_{h}\right)+\partial_{x} q_{0}\left(u_{h}\right)$ are compact in $H_{l o c}^{-1}$;

(3) For any $C^{2}$ convex entropy pair $(\eta(u), q(u)), \partial_{t} \eta\left(u_{h}\right)+\partial_{x} q\left(u_{h}\right) \leq o(1)$ in $\mathcal{D}^{\prime}$, provided that $|\eta(u)|+|q(u)| \leq M\left(1+|u|^{r}\right), r<p$.

Then there is a subsequence (still denoted as) $u_{h}$ such that $u_{h}(x, t) \rightarrow u(x, t)$ a.e. as $h \rightarrow 0$ and $u$ is the entropy solution of (1.1) satisfying $\partial_{t} \eta(u)+\partial_{x} q(u) \leq 0 \quad$ in $\mathcal{D}^{\prime}$ for any $C^{2}$ convex entropy pair $(\eta, q)$. 
We remark that, for general conservation laws, the conditions (1) and (2) imply that $\mathrm{w}-\lim f\left(u_{h}\right)=f\left(\mathrm{w}-\lim u_{h}\right) \quad$ in $L^{2}$ and the condition (3) implies the following entropy condition for the Young measures $\nu_{x, t}$, determined by the Lax-Wendroff approximate solutions $u_{h}(x, t)$,

$$
\partial_{t}\left\langle\nu_{x, t}, \eta\right\rangle+\partial_{x}\left\langle\nu_{x, t}, q\right\rangle \leq 0
$$

in the sense of distributions. We refer the reader to Chen-Lu [3] for a detailed discussion. The proof of the $L^{\infty}$ version of Theorem 2.1 can be found in [2], [3], [28] using the div-curl lemma of Tartar and Murat [28].

For a $2 \times 2$ system of conservation laws endowed with global Riemann invariants, we have the following similar framework.

Theorem 2.2. Let $u_{h}(x, t)$ be numerical approximate solutions of the $2 \times 2$ genuinely nonlinear and strictly hyperbolic system of conservation laws (1.1) satisfying the following conditions:

(1) $u_{h}$ is bounded in $L^{\infty}$;

(2) For any $C^{2}$ entropy pair $(\eta, q), \partial_{t} \eta\left(u_{h}\right)+\partial_{x} q\left(u_{h}\right)$ is compact in $H_{l o c}^{-1}$;

(3) For any $C^{2}$ convex entropy pair $(\eta, q), \partial_{t} \eta\left(u_{h}\right)+\partial_{x} q\left(u_{h}\right) \leq o(1)$ in $\mathcal{D}^{\prime}$.

Then there is a subsequence (still denoted as) $u_{h}$ such that $u_{h}(x, t) \rightarrow u(x, t)$ a.e. as $h \rightarrow 0$ and $u$ is the entropy solution of (1.1) satisfying $\partial_{t} \eta(u)+\partial_{x} q(u) \leq 0$ in $\mathcal{D}^{\prime}$ for any $C^{2}$ convex entropy $(\eta, q)$.

This theorem is proved by DiPerna [5] by estimating the entropy dissipation measures and by using the Lax entropy pairs and the compensated compactness method. An alternative extension proof can be found in [16], [23]. Finally we state the following two lemmas, which can be found in [17] and [2], respectively.

Lemma 2.1. The embedding of the positive cone of $W^{-1, p}$ in $W_{l o c}^{-1, q}$ is completely continuous for all $q<p$.

Lemma 2.2. Let $\Omega \subset R^{n}$ be a bounded open set. Then

$$
\begin{aligned}
& \text { (compact set of } \left.W_{\text {loc }}^{-1, q}(\Omega)\right) \cap\left(\text { bounded set of } W_{l o c}^{-1, r}(\Omega)\right) \\
& \subset\left(\text { compact set of } H_{l o c}^{-1}(\Omega)\right),
\end{aligned}
$$

where $q$ and $r$ are constants, $1<q \leq 2<r<\infty$.

\section{Convex scalar COnservation laWs}

In this section we are concerned with the Lax-Wendroff approximate solutions of the scalar conservation laws (1.1) with flux function $f(u)$ satisfying

$$
f^{\prime \prime}(u) \geq c_{0}>0, \quad f^{(k)}(u) \sim O\left(|u|^{m-k}\right), \quad \text { for }|u| \gg 1, k=0,1,2 .
$$

For convenience, we write the Lax-Wendroff scheme (1.2) into the form

$$
u_{j}^{n+1}=u_{j}^{n}+F_{j}^{n}+H_{j}^{n}+J_{j}^{n},
$$

where

$$
\begin{gathered}
F_{j}^{n}=-\frac{1}{2} \lambda_{n} \Delta_{0} f\left(u_{j}^{n}\right), \quad H_{j}^{n}=\frac{1}{2} \lambda_{n}^{2} \Delta_{-}\left(\frac{\left(\Delta_{+} f\left(u_{j}^{n}\right)\right)^{2}}{\Delta_{+} u_{j}^{n}}\right), \\
J_{j}^{n}=\lambda_{n} \Delta_{-}\left(\tilde{\beta}_{j+1 / 2}^{n}\left|\Delta_{+} a\left(u_{j}^{n}\right)\right| \Delta_{+} u_{j}^{n}\right),
\end{gathered}
$$


with

$$
\tilde{\beta}_{j+1 / 2}^{n}=\beta_{j+1 / 2}^{n}+\lambda_{n} \frac{a\left(\frac{u_{j}^{n}+u_{j+1}^{n}}{2}\right)-\Delta_{+} f\left(u_{j}^{n}\right) / \Delta_{+} u_{j}^{n}}{\left|\Delta_{+} a\left(u_{j}^{n}\right)\right|}=\beta_{j+1 / 2}^{n}+O\left(\lambda_{n}\left|a^{\prime}\right|\right) .
$$

From $u_{j}^{n}$, we construct the Lax-Wendroff approximate solutions on $\mathbb{R}_{+}^{2}$ :

$$
u_{h}(x, t) \equiv u_{j}^{n}, \quad \text { for }(x, t) \in\left[x_{j-1 / 2}, x_{j+1 / 2}\right) \times\left[t_{n}, t_{n+1}\right) .
$$

Then we have the following main convergence theorem of this section.

Theorem 3.1. Let $u_{h}(x, t)$ be the Lax-Wendroff approximate solutions (3.4) of the scalar conservation laws (1.1) and (3.1). Assume that the coefficient $\beta_{0}>0$ is suitably large and the CFL number $\varepsilon_{0}$ given by (1.3) is suitably small. Then there exists a subsequence strongly converging to the entropy solution of (1.1) satisfying the following entropy condition $\partial_{t} \eta(u)+\partial_{x} q(u) \leq 0$ in $\mathcal{D}^{\prime}$ for any $C^{2}$ convex entropy pair $(\eta, q)$ satisfying $\eta^{(k)}(u) \sim O\left(|u|^{r-k}\right)$, for $|u| \gg 1,0 \leq k \leq r<4 m$.

The proof consists of the following three subsections in which we check the three conditions in Theorem 2.1, respectively. For simplicity of our proof, we drop the subscript $n$ and use the following notations:

$$
\begin{gathered}
f_{j}=f\left(u_{j}^{n}\right), \quad a_{j}=a\left(u_{j}^{n}\right), \quad a_{j+1 / 2}=a\left(\frac{u_{j}^{n}+u_{j+1}^{n}}{2}\right), \\
\eta_{j}=\eta\left(u_{j}^{n}\right), \quad \eta_{j}^{\prime}=\eta^{\prime}\left(u_{j}^{n}\right), \quad \eta_{j}^{\prime \prime}=\eta^{\prime \prime}\left(u_{j}^{n}\right),
\end{gathered}
$$

where $\eta$ is an entropy function of (1.1). We first introduce the following three technical lemmas.

Lemma 3.1. If $g^{\prime}(u) \geq c_{0}>0$ and $g^{(k)}(u)=c_{k} u^{m-k}(1+o(1)),|u| \gg 1$, for some constants $c_{k}, k=0,1,2$, then there is $\bar{u} \gg 1$ and $\alpha>0$ such that

$$
\frac{g(u)-g(v)}{u-v} \geq \Phi(\max (|u|,|v|)), \quad \text { with } \Phi(s)= \begin{cases}\alpha g^{\prime}(s), & s \geq \bar{u}, \\ c_{0}, & s<\bar{u} .\end{cases}
$$

The proof is straightforward and hence is omitted.

Summing by parts, one has

Lemma 3.2. If $\left\{a_{j}\right\}$ and $\left\{b_{j}\right\}$ are two arbitrary sequences, then

$$
\Delta_{+}\left(a_{j} \Delta_{0} b_{j}\right)=\Delta_{0}\left(a_{j} \Delta_{+} b_{j}\right)+\Delta_{-}\left(\Delta_{+} a_{j} \Delta_{+} b_{j}\right),
$$

and

$$
2 \sum_{j} a_{j} b_{j} \Delta_{0} b_{j}=\sum_{j}\left(\Delta_{+} b_{j}\right)^{2} \Delta_{+} a_{j}-\sum_{j} b_{j}^{2} \Delta_{0} a_{j},
$$

provided that the above sums make sense.

Proof. The formula (3.6) comes from a direct computation. Notice that

$$
\Delta_{0}\left(a_{j} b_{j}\right)=a_{j} \Delta_{0} b_{j}+b_{j} \Delta_{0} a_{j}+\Delta_{-}\left(\Delta_{+} a_{j} \Delta_{+} b_{j}\right) .
$$

Multiplying both sides of the above equation by $b_{j}$, taking sum for $j$, and summing by parts, one arrives at (3.7).

Using the relation $\eta^{\prime} f^{\prime}=q^{\prime}$, expanding $\eta$ at $u_{j}$ in the intervals $\left[u_{j-1}, u_{j}\right]$ and $\left[u_{j}, u_{j+1}\right]$, and using the integration by parts, one has 
Lemma 3.3. If $(\eta, q)$ is an entropy pair of (1.1), then

$$
\begin{aligned}
\eta_{j}^{\prime} \Delta_{0} f_{j}= & \Delta_{0} q_{j}-\frac{1}{2} \eta_{j}^{\prime \prime}\left(\Delta_{+} u_{j}\right)^{2} \Delta_{+} a_{j}-\frac{1}{2} \eta_{j}^{\prime \prime} \Delta_{-}\left(\left(\Delta_{+} u_{j}\right)^{2} a_{j}\right) \\
& +\frac{1}{2} \eta_{j}^{\prime \prime} \int_{u_{j-1}}^{u_{j+1}}\left(u_{j}-s\right)^{2} a^{\prime}(s) d s \\
& +\frac{1}{2} \eta^{\prime \prime \prime}\left(\xi_{j}\right) \int_{u_{j}}^{u_{j+1}}\left(u_{j}-s\right)^{2} a(s) d s+\frac{1}{2} \eta^{\prime \prime \prime}\left(\xi_{j-1}\right) \int_{u_{j-1}}^{u_{j}}\left(u_{j}-s\right)^{2} a(s) d s,
\end{aligned}
$$

where $\xi_{j-1}$ and $\xi_{j}$ are some values in $\left[u_{j-1}, u_{j}\right]$ and $\left[u_{j}, u_{j+1}\right]$, respectively.

Proof. Using the relation $\eta^{\prime} f^{\prime}=q^{\prime}$ and expanding $\eta$ at $u_{j}$ in the intervals $\left[u_{j-1}, u_{j}\right]$ and $\left[u_{j}, u_{j+1}\right]$, one has

$$
\begin{aligned}
\eta_{j}^{\prime} \Delta_{0} f_{j}= & \Delta_{0} q_{j}+\eta_{j}^{\prime \prime} \int_{u_{j-1}}^{u_{j+1}}\left(u_{j}-s\right) a(s) d s \\
& +\frac{1}{2} \eta^{\prime \prime \prime}\left(\xi_{j}\right) \int_{u_{j}}^{u_{j+1}}\left(u_{j}-s\right)^{2} a(s) d s+\frac{1}{2} \eta^{\prime \prime \prime}\left(\xi_{j-1}\right) \int_{u_{j-1}}^{u_{j}}\left(u_{j}-s\right)^{2} a(s) d s,
\end{aligned}
$$

for some $\xi_{j} \in\left[u_{j}, u_{j+1}\right]$ and $\xi_{j-1} \in\left[u_{j-1}, u_{j}\right]$. Now using the integration by parts to the second term on the right-hand side of the above equation, we obtain the lemma.

We remark here that we split the remainders in Lemma 3.3 into two cells $\left[u_{j-1}, u_{j}\right]$ and $\left[u_{j}, u_{j+1}\right]$. A basic reason for this is that we do not require the $L^{\infty}$ bound of the approximate solutions, and thus need to control the growth rate. This kind of splitting techniques will be used in several places in this section.

3.1. $L^{p}$ estimate. We now verify the first condition of Theorem 2.1 for the LaxWendroff scheme (3.2)-(3.3). Since the flux function has the algebraic growth rate $m$, we only need to show that $u_{h}$ is bounded in $L^{2}$ and $L^{4 m-2}$. The method used here is to estimate the entropy function $\eta$ with certain required growth rate. In order to control the growth rate in the compactness analysis in Subsection 3.3, we estimate the entropy with a growth rate slightly more than that required in this subsection. For this reason, we choose a strictly convex entropy function

$$
\eta(u)=\frac{1}{4 m(4 m-1)} u^{4 m}+\frac{1}{2} u^{2}
$$

and estimate the bound of $\sum \eta\left(u_{j}^{n}\right)$ for the Lax-Wendroff scheme $u_{j}^{n}$.

We first expand the time increment of $\sum \eta\left(u_{j}^{n}\right)$ and split it into three terms:

$$
\begin{aligned}
\sum_{j}\left(\eta\left(u_{j}^{n+1}\right)-\eta\left(u_{j}^{n}\right)\right)= & \sum_{j} \eta^{\prime}\left(u_{j}^{n}\right)\left(u_{j}^{n+1}-u_{j}^{n}\right)+\frac{1}{2} \sum_{j} \eta^{\prime \prime}\left(u_{j}^{n}\right)\left(u_{j}^{n+1}-u_{j}^{n}\right)^{2} \\
& +\sum_{k=3}^{4 m} \frac{1}{k !} \sum_{j} \eta^{(k)}\left(u_{j}^{n}\right)\left(u_{j}^{n+1}-u_{j}^{n}\right)^{k} \equiv I_{1}+I_{2}+I_{3},
\end{aligned}
$$

which will be estimated in this subsection, where we used the exact expansion to the highest order to avoid the remainder in $\left[u_{j}^{n}, u_{j}^{n+1}\right]$, which is difficult to control. 
Estimate of $I_{1}$. Plugging (3.2) into $I_{1}$, one has

$$
I_{1}=\sum_{j} \eta_{j}^{\prime} J_{j}+\sum_{j} \eta_{j}^{\prime} H_{j}+\sum_{j} \eta_{j}^{\prime} F_{j}
$$

To estimate the first term, one uses the expression of $J_{j}$ in (3.3), the summation by parts, and Lemma 3.1 to get

$$
\sum_{j} \eta_{j}^{\prime} J_{j}=-\lambda \sum_{j} \tilde{\beta}_{j+1 / 2}^{n} \Delta_{+} \eta_{j}^{\prime}\left|\Delta_{+} a_{j}\right| \Delta_{+} u_{j} \leq-\lambda \beta_{0} \sum_{j} \Phi\left(s_{j}\right)\left|\Delta_{+} u_{j}\right|^{3},
$$

where

$$
s_{j}=\max \left(\left|u_{j}\right|,\left|u_{j+1}\right|\right), \quad \Phi(s)= \begin{cases}c s^{5 m-4}, & s \geq \bar{u}, \\ c_{0}, & s<\bar{u},\end{cases}
$$

for some constant $c>0$. Note that (3.10) is the sum of products of three first-order differences due to the fact that the viscosity term is second-order accurate. This is the main dissipative term that is used to control all of the error terms.

In estimating the second term, one uses the summation by parts and the expression of $H_{j}$ in (3.3), and substitutes the expansion

$$
\Delta_{+} \eta_{j}^{\prime}=\eta_{j}^{\prime \prime} \Delta_{+} u_{j}+\frac{1}{2} \eta^{\prime \prime \prime}\left(\xi_{j}\right)\left(\Delta_{+} u_{j}\right)^{2},
$$

in the resulting terms to get

$$
\begin{aligned}
\sum_{j} \eta_{j}^{\prime} H_{j} & =-\frac{1}{2} \lambda^{2} \sum_{j} \frac{\left(\Delta_{+} f_{j}\right)^{2}}{\Delta_{+} u_{j}} \Delta_{+} \eta_{j}^{\prime} \\
& =-\frac{1}{2} \lambda^{2} \sum_{j} \eta_{j}^{\prime \prime}\left(\Delta_{+} f_{j}\right)^{2}-\frac{1}{4} \lambda^{2} \sum_{j} \eta^{\prime \prime \prime}\left(\xi_{j}\right)\left(\Delta_{+} f_{j}\right)^{2} \Delta_{+} u_{j}
\end{aligned}
$$

We use the term of the right-hand side in (3.10) to control the second term in (3.13). Notice that there is a factor $\left(\Delta_{+} u_{j}\right)^{3}$ in the second term that can be clearly controlled by the term of the right-hand side in (3.10). The factor in front of the difference is of growth rate $2(m-1)+4 m-4=6(m-1)$ that is larger than $5 m-4$ in (3.11). Noting that there is also a parameter $\lambda^{2}$ in front of this factor, therefore, we can use the fact $\lambda\left|\Delta_{+} f_{j} / \Delta_{+} u_{j}\right| \leq \varepsilon_{0}$ to control certain powers of the growth rate to obtain

$$
\sum_{j} \eta_{j}^{\prime} H_{j} \leq-\frac{1}{2} \lambda^{2} \sum_{j} \eta_{j}^{\prime \prime}\left(\Delta_{+} f_{j}\right)^{2}+\lambda \varepsilon_{0} C \sum_{j} \Phi\left(s_{j}\right)\left|\Delta_{+} u_{j}\right|^{3}
$$

for some positive constant $C>0$. This kind of simple techniques will be used in several places in the rest of this section and we will omit the detailed explanations.

Here and henceforth, we use the notations $\xi_{j}$ and $\bar{\xi}_{j}$ as some values in $\left[u_{j}, u_{j+1}\right]$, and $C$ is a positive constant, independent of the grid size $h$, the CFL number, the viscosity constant $\beta$, and the numerical solution $u_{h}$. In different contexts, they may have different values. 
Now we estimate the term $\sum_{j} \eta_{j}^{\prime} F_{j}$. Writing the expression of $F_{j}$ in (3.3) in the integral form and using the conservative property of entropy, one has

$$
\begin{aligned}
\sum_{j} \eta_{j}^{\prime} F_{j}= & -\frac{1}{2} \lambda \sum_{j} \eta^{\prime}\left(u_{j}\right) \int_{u_{j-1}}^{u_{j+1}} a(s) d s \\
= & \frac{1}{2} \lambda \sum_{j} \int_{u_{j-1}}^{u_{j+1}}\left(\eta^{\prime}(s)-\eta^{\prime}\left(u_{j}\right)\right) a(s) d s \\
= & \frac{1}{2} \lambda \sum_{j} \eta^{\prime \prime}\left(u_{j}\right) \int_{u_{j-1}}^{u_{j+1}}\left(s-u_{j}\right) a(s) d s \\
& +\frac{1}{4} \lambda \sum_{j} \eta^{\prime \prime \prime}\left(\xi_{j-1}\right) \int_{u_{j-1}}^{u_{j}}\left(s-u_{j}\right)^{2} a(s) d s \\
& +\frac{1}{4} \lambda \sum_{j} \eta^{\prime \prime \prime}\left(\xi_{j}\right) \int_{u_{j}}^{u_{j+1}}\left(s-u_{j}\right)^{2} a(s) d s .
\end{aligned}
$$

Applying the following integration by parts to the first term in the right-hand side of the above equality

$$
\int_{u_{j-1}}^{u_{j+1}}\left(s-u_{j}\right) a(s) d s=\frac{1}{2}\left(\Delta_{+} u_{j}\right)^{2} \Delta_{+} a_{j}-\frac{1}{2} \int_{u_{j-1}}^{u_{j+1}}\left(s-u_{j}\right)^{2} a^{\prime}(s) d s,
$$

and plugging the above two estimates back into (3.15), we obtain

$$
\sum_{j} \eta_{j}^{\prime} F_{j} \leq \lambda C \sum_{j} \Phi\left(s_{j}\right)\left|\Delta_{+} u_{j}\right|^{3} .
$$

Finally, one uses (3.10), (3.14), and (3.16) to get the following estimate:

$I_{1} \leq-\lambda \beta_{0} \sum_{j} \Phi\left(s_{j}\right)\left|\Delta_{+} u_{j}\right|^{3}-\frac{1}{2} \lambda^{2} \sum_{j} \eta_{j}^{\prime \prime}\left(\Delta_{+} f_{j}\right)^{2}+\lambda\left(1+\varepsilon_{0}\right) C \sum_{j} \Phi\left(s_{j}\right)\left|\Delta_{+} u_{j}\right|^{3}$.

Estimate of $I_{2}$. Substituting (3.2) into $I_{2}$ in (3.8), one has the expansion

$$
I_{2}=\frac{1}{2} \sum_{j} \eta^{\prime \prime}\left(u_{j}\right)\left(F_{j}^{2}+H_{j}^{2}+2 F_{j} H_{j}+J_{j}^{2}+2 J_{j}\left(F_{j}+H_{j}\right)\right) .
$$

We now estimate $I_{2}$ term by term. In estimating the first term in (3.18), one uses the identity

$$
\left(\Delta_{0} f_{j}\right)^{2}=2\left(\Delta_{+} f_{j}\right)^{2}+2\left(\Delta_{+} f_{j-1}\right)^{2}-\left(\Delta_{-} \Delta_{+} f_{j}\right)^{2},
$$

and a direct estimate of $F_{j}$ from its expression in (3.3) to get

$$
\begin{aligned}
\sum_{j} \eta_{j}^{\prime \prime} F_{j}^{2} & =\frac{1}{2} \lambda^{2} \sum_{j} \eta_{j}^{\prime \prime}\left(\left(\Delta_{+} f_{j}\right)^{2}+\left(\Delta_{+} f_{j-1}\right)^{2}\right)-\frac{1}{4} \lambda^{2} \sum_{j} \eta^{\prime \prime}\left(u_{j}\right)\left(\Delta_{-} \Delta_{+} f_{j}\right)^{2} \\
& =\lambda^{2} \sum_{j} \eta_{j}^{\prime \prime}\left(\Delta_{+} f_{j}\right)^{2}-\frac{1}{4} \lambda^{2} \sum_{j} \eta_{j}^{\prime \prime}\left(\Delta_{-} \Delta_{+} f_{j}\right)^{2}+\frac{1}{2} \lambda^{2} \sum_{j} \Delta_{+} \eta_{j}^{\prime \prime}\left(\Delta_{+} f_{j}\right)^{2} .
\end{aligned}
$$


In the first and third terms on the right-hand side of the above inequality, one uses the Taylor expansion for $\Delta_{+} f_{j}$ and some simple calculations used in estimating (3.13) to obtain

$$
\begin{aligned}
\sum_{j} \eta_{j}^{\prime \prime} F_{j}^{2} \leq & \lambda^{2} \sum_{j} \eta_{j}^{\prime \prime} a_{j+1 / 2}^{2}\left(\Delta_{+} u_{j}\right)^{2}-\frac{1}{4} \lambda^{2} \sum_{j} \eta_{j}^{\prime \prime}\left(\Delta_{-} \Delta_{+} f_{j}\right)^{2} \\
& +\lambda \varepsilon_{0} C \sum_{j} \Phi\left(s_{j}\right)\left|\Delta_{+} u_{j}\right|^{3} .
\end{aligned}
$$

Notice that the first term on the right-hand side of (3.20) is exactly the same as the second term on that of (3.17) except with a different sign, which cancel each other. The second term on the right-hand side of (3.20) is a sum of squares of second-order differences in a favorable sign. We will use it to control a similar term below.

To estimate the second term in (3.18), we use the identity

$$
\Delta_{-}\left(\frac{\left(\Delta_{+} f_{j}\right)^{2}}{\Delta_{+} u_{j}}\right)=\Delta_{-} \Delta_{+} f_{j} \frac{\Delta_{+} f_{j-1}}{\Delta_{+} u_{j-1}}+\Delta_{+} f_{j} \Delta_{-}\left(\frac{\Delta_{+} f_{j}}{\Delta_{+} u_{j}}\right)
$$

and directly estimate the expression of $H_{j}$ in (3.3) to get

$$
\begin{aligned}
\sum_{j} \eta_{j}^{\prime \prime} H_{j}^{2} \leq & \frac{1}{2} \lambda^{4} \sum_{j} \eta_{j}^{\prime \prime}\left(\Delta_{-} \Delta_{+} f_{j}\right)^{2}\left(\frac{\Delta_{+} f_{j-1}}{\Delta_{+} u_{j-1}}\right)^{2} \\
& +\frac{1}{2} \lambda^{4} \sum_{j} \eta_{j}^{\prime \prime}\left(\Delta_{+} f_{j}\right)^{2}\left(\Delta_{-}\left(\frac{\Delta_{+} f_{j}}{\Delta_{+} u_{j}}\right)\right)^{2} .
\end{aligned}
$$

The first term on the right-hand side of the above inequality can be controlled by the second term on the right-hand side of (3.20) in view of the fact that the CFL number is less than 1 . The last term of the above inequality involves three points. Hence we split it into the following two terms by using the Taylor expansion for $a$ in the intervals $\left[u_{j-1}, u_{j}\right]$ and $\left[u_{j}, u_{j+1}\right]$ :

$$
\begin{aligned}
\lambda^{4} \sum_{j} \eta_{j}^{\prime \prime}\left(\Delta_{+} f_{j}\right)^{2}\left(\Delta_{-}\left(\frac{\Delta_{+} f_{j}}{\Delta_{+} u_{j}}\right)\right)^{2} \leq & 2 \lambda \varepsilon_{0}^{3} \sum_{j} \eta_{j}^{\prime \prime}\left(\Delta_{+} u_{j}\right)^{2}\left(\Delta_{-}\left(\frac{\Delta_{+} f_{j}}{\Delta_{+} u_{j}}\right)\right)^{2} \\
\leq & \lambda \varepsilon_{0}^{3} \sum_{j} \eta_{j}^{\prime \prime}\left|a^{\prime}\left(\xi_{j-1}\right)\right|\left|\Delta_{-} u_{j}\right|\left(\Delta_{+} u_{j}\right)^{2} \\
& +\lambda \varepsilon_{0}^{3} \sum_{j} \eta_{j}^{\prime \prime}\left|a^{\prime}\left(\xi_{j}\right) \| \Delta_{+} u_{j}\right|\left(\Delta_{+} u_{j}\right)^{2}
\end{aligned}
$$

The last term only involves two points and hence can be directly estimated. In the last second term, we use the Hölder inequality to split it again to obtain

$$
\begin{aligned}
\sum_{j} \eta_{j}^{\prime \prime}\left|a^{\prime}\left(\xi_{j-1}\right)\right|\left|\Delta_{-} u_{j}\right|\left(\Delta_{+} u_{j}\right)^{2} & \leq \sum_{j}\left|u_{j}\right|^{4 m-2}\left|s_{j-1}\right|^{m-2}\left|\Delta_{-} u_{j}\right|\left(\Delta_{+} u_{j}\right)^{2} \\
& \leq C \sum_{j} \Phi\left(s_{j}\right)\left|\Delta_{+} u_{j}\right|^{3}
\end{aligned}
$$


The methods used here will be applied to estimating the similar terms. With the above three estimates, we arrive at

$$
\sum_{j} \eta_{j}^{\prime \prime} H_{j}^{2} \leq \frac{1}{2} \lambda^{4} \sum_{j} \eta_{j}^{\prime \prime}\left(\Delta_{-} \Delta_{+} f_{j}\right)^{2}\left(\frac{\Delta_{+} f_{j-1}}{\Delta_{+} u_{j-1}}\right)^{2}+\lambda \varepsilon_{0}^{3} C \sum_{j} \Phi\left(s_{j}\right)\left|\Delta_{+} u_{j}\right|^{3} .
$$

The third term in (3.18) consists of products of a factor of first-order difference and a factor of second-order difference, we utilize a symmetric property to transform it into a sum of products of three differences. In doing this, we first split the positive function $\eta^{\prime \prime}$, and sum by parts to get

$$
\begin{aligned}
-\frac{1}{4} \lambda^{3} & \sum_{j} \eta_{j}^{\prime \prime} \Delta_{0} f_{j} \Delta_{-}\left(\frac{\left(\Delta_{+} f_{j}\right)^{2}}{\Delta_{+} u_{j}}\right) \\
= & -\frac{1}{4} \lambda^{3} \sum_{j} \sqrt{\eta_{j}^{\prime \prime}} \Delta_{0} f_{j}\left[\Delta_{-}\left(\sqrt{\eta_{j}^{\prime \prime}} \frac{\left(\Delta_{+} f_{j}\right)^{2}}{\Delta_{+} u_{j}}\right)-\Delta_{-} \sqrt{\eta_{j}^{\prime \prime}} \frac{\left(\Delta_{+} f_{j-1}\right)^{2}}{\Delta_{+} u_{j-1}}\right] \\
= & \frac{1}{4} \lambda^{3} \sum_{j}\left(\sqrt{\eta_{j}^{\prime \prime}} \Delta_{+} f_{j}\right) \Delta_{+}\left(\sqrt{\eta_{j}^{\prime \prime}} \Delta_{0} f_{j} \frac{\Delta_{+} f_{j}}{\Delta_{+} u_{j}}\right) \\
& +\frac{1}{4} \lambda^{3} \sum_{j} \sqrt{\eta_{j}^{\prime \prime}} \Delta_{0} f_{j} \Delta_{-} \sqrt{\eta_{j}^{\prime \prime}} \frac{\left(\Delta_{+} f_{j-1}\right)^{2}}{\Delta_{+} u_{j-1}}
\end{aligned}
$$

Now the last term consists of products of three differences and hence can be directly estimated. The first term has some symmetric property after switching the difference operator $\Delta_{+}$with $\Delta_{0}$. This can be done by using Lemma 3.2 as follows.

$$
\begin{aligned}
\sum_{j}\left(\sqrt{\eta_{j}^{\prime \prime}} \Delta_{+} f_{j}\right) \Delta_{+}\left(\sqrt{\eta_{j}^{\prime \prime}} \Delta_{0} f_{j}\right) \frac{\Delta_{+} f_{j}}{\Delta_{+} u_{j}} & =\sum_{j}\left(\sqrt{\eta_{j}^{\prime \prime}} \Delta_{+} f_{j}\right) \Delta_{0}\left(\sqrt{\eta_{j}^{\prime \prime}} \Delta_{+} f_{j}\right) \frac{\Delta_{+} f_{j}}{\Delta_{+} u_{j}} \\
& +\sum_{j}\left(\sqrt{\eta_{j}^{\prime \prime}} \Delta_{+} f_{j}\right) \Delta_{-}\left(\Delta_{+} \sqrt{\eta_{j}^{\prime \prime}} \Delta_{+} f_{j}\right) \frac{\Delta_{+} f_{j}}{\Delta_{+} u_{j}}
\end{aligned}
$$

Again the last term in the above equality is a sum of products of three differences in $u_{j}$ and hence can be estimated directly. The first term can now be transformed into a sum of products of three differences by using Lemma 3.3 where $b_{j}$ are replaced by $\sqrt{\eta_{j}^{\prime \prime}} \Delta_{+} f_{j}$.

$$
\begin{aligned}
\sum_{j}\left(\sqrt{\eta_{j}^{\prime \prime}} \Delta_{+} f_{j}\right) \Delta_{0}\left(\sqrt{\eta_{j}^{\prime \prime}} \Delta_{+} f_{j}\right) \frac{\Delta_{+} f_{j}}{\Delta_{+} u_{j}} & =\frac{1}{2} \sum_{j}\left(\Delta_{+}\left(\sqrt{\eta_{j}^{\prime \prime}} \Delta_{+} f_{j}\right)\right)^{2} \Delta_{+}\left(\frac{\Delta_{+} f_{j}}{\Delta_{+} u_{j}}\right) \\
& -\frac{1}{2} \sum_{j}\left(\sqrt{\eta_{j}^{\prime \prime}} \Delta_{+} f_{j}\right)^{2} \Delta_{0}\left(\frac{\Delta_{+} f_{j}}{\Delta_{+} u_{j}}\right) .
\end{aligned}
$$

Finally, combining all the estimates (3.23)-(3.24), we arrive at

$$
\sum_{j} \eta_{j}^{\prime \prime} F_{j} H_{j} \leq \lambda \varepsilon_{0}^{2} C \sum_{j} \Phi\left(s_{j}\right)\left|\Delta_{+} u_{j}\right|^{3} .
$$


Estimating the remainder three terms in (3.18) is rather simple since each of them consists of more than three difference factors. We estimate them as follows:

$$
\sum_{j} \eta_{j}^{\prime \prime} J_{j}^{2}=\lambda^{2} \sum_{j} \eta_{j}^{\prime \prime}\left(\Delta_{-}\left(\tilde{\beta}_{j+1 / 2}^{n}\left|\Delta_{+} a_{j}\right| \Delta_{+} u_{j}\right)\right)^{2} \leq \lambda \varepsilon_{0} \beta_{1}^{2} C \sum_{j} \Phi\left(s_{j}\right)\left|\Delta_{+} u_{j}\right|^{3},
$$

and

$$
\begin{aligned}
2 \sum_{j} \eta_{j}^{\prime \prime} J_{j}\left(F_{j}+H_{j}\right)= & -\lambda^{2} \sum_{j} \eta_{j}^{\prime \prime} \Delta_{-}\left(\tilde{\beta}_{j+1 / 2}^{n}\left|\Delta_{+} a_{j}\right| \Delta_{+} u_{j}\right) \Delta_{0} f_{j} \\
& +\lambda^{3} \sum_{j} \eta_{j}^{\prime \prime} \Delta_{-}\left(\tilde{\beta}_{j+1 / 2}^{n}\left|\Delta_{+} a_{j}\right| \Delta_{+} u_{j}\right) \Delta_{-}\left(a_{j+1 / 2} \Delta_{+} f_{j}\right) \\
\leq & \lambda\left(\varepsilon_{0}+\varepsilon_{0}^{2}\right) \beta_{1} C \sum_{j} \Phi\left(s_{j}\right)\left|\Delta_{+} u_{j}\right|^{3} .
\end{aligned}
$$

The estimate of $I_{2}$ is now completed by combining (3.20) and (3.22) with (3.25)(3.27).

$$
\begin{aligned}
I_{2} \leq & \frac{1}{2} \lambda^{2} \sum_{j} \eta_{j}^{\prime \prime} a_{j+1 / 2}^{2}\left(\Delta_{+} u_{j}\right)^{2}-\frac{1}{8} \lambda^{2} \sum_{j}\left(1-2 \lambda^{2} a_{j-1 / 2}^{2}\right) \eta_{j}^{\prime \prime}\left(\Delta_{-} \Delta_{+} f_{j}\right)^{2} \\
& +\lambda \varepsilon_{0}\left(1+\varepsilon_{0}+\varepsilon_{0}^{2}+\beta_{1}+\beta_{1}^{2}\right) C \sum_{j} \Phi\left(s_{j}\right)\left|\Delta_{+} u_{j}\right|^{3} .
\end{aligned}
$$

Estimate of $I_{3}$. The estimate of $I_{3}$ is rather easy. We first substitute the expansion of $u_{j}^{n+1}-u_{j}^{n}$ in (3.2)-(3.3) into $I_{3}$ to get

$$
\left|I_{3}\right| \leq \sum_{k=3}^{4 m} \frac{3^{k}}{k !} \sum_{j}\left|\eta^{(k)}\left(u_{j}^{n}\right)\right|\left(\left|F_{j}\right|^{k}+\left|H_{j}\right|^{k}+\left|J_{j}\right|^{k}\right) .
$$

Using the expressions in (3.3), we have

$$
\begin{aligned}
\left|I_{3}\right| \leq \sum_{k=3}^{4 m} & \frac{3^{k}}{k !} \sum_{j}\left|\eta^{(k)}\left(u_{j}^{n}\right)\right|\left(\lambda^{k}\left|\Delta_{+} f_{j}\right|^{k}+\lambda^{k}\left|\Delta_{-} f_{j}\right|^{k}+\lambda^{2 k}\left|a_{j+1 / 2} \Delta_{+} f_{j}\right|^{k}\right. \\
& \left.\quad+\lambda^{2 k}\left|a_{j-1 / 2} \Delta_{-} f_{j}\right|^{k}+\left(2 \lambda \beta_{1}\right)^{k}\left|\Delta_{+} a_{j} \Delta_{+} u_{j}\right|^{k}+\left(2 \lambda \beta_{1}\right)^{k}\left|\Delta_{-} a_{j} \Delta_{-} u_{j}\right|^{k}\right) \\
\leq & \lambda C \sum_{k=3}^{4 m}\left(\varepsilon_{0}^{k-1}+\varepsilon_{0}^{2 k-1}+\varepsilon_{0}^{k-1} \beta_{1}^{k}\right) \sum_{j} \Phi\left(s_{j}\right)\left|\Delta_{+} u_{j}\right|^{3} .
\end{aligned}
$$

We have now estimated all $I_{1}, I_{2}$, and $I_{3}$. Using (3.17), (3.28), and (3.30), we obtain

$$
\begin{aligned}
\sum_{j}\left(\eta_{j}^{n+1}-\eta_{j}^{n}\right) \leq & -\frac{\lambda}{2} \beta_{0} \sum_{j} \Phi\left(s_{j}\right)\left|\Delta_{+} a_{j}\right|^{3}-\frac{1}{8} \lambda^{2}\left(1-2 \varepsilon_{0}^{2}\right) \sum_{j} \eta_{j}^{\prime \prime}\left(\Delta_{-} \Delta_{+} f_{j}\right)^{2} \\
& +\lambda C\left(1+\varepsilon_{0} \beta_{1}+\sum_{k=2}^{4 m} \varepsilon_{0}^{k-1} \beta_{1}^{k}\right) \sum_{j} \Phi\left(s_{j}\right)\left|\Delta_{+} u_{j}\right|^{3}
\end{aligned}
$$


Choosing $\beta_{0}$ large and $\varepsilon_{0} \beta_{1}$ small, one has

$$
\sum_{j}\left(\eta_{j}^{n+1}-\eta_{j}^{n}\right) \leq-\lambda C_{1} \sum_{j} \Phi\left(s_{j}\right)\left|\Delta_{+} a_{j}\right|^{3}-\lambda^{2} C_{2} \sum_{j}\left(\Delta_{-} \Delta_{+} f_{j}\right)^{2} .
$$

This gives the following proposition.

Proposition 3.1. Under the same assumptions of Theorem 3.1, we have

$$
\sum_{j} \eta\left(u_{j}^{n}\right) h+\lambda \sum_{k \leq n ; j} \Phi\left(s_{j}^{k}\right)\left|\Delta_{+} u_{j}^{k}\right|^{3} h+\lambda^{2} \sum_{k \leq n ; j}\left(\Delta_{-} \Delta_{+} f\left(u_{j}^{k}\right)\right)^{2} h \leq C,
$$

where $\eta$ is the entropy function given by (3.7).

As an immediate consequence, we have

Corollary 3.1. Under the same assumptions of Theorem 3.1, we have

$$
\left\|u_{h}\right\|_{L_{l o c}^{4 m}}+\left\|\left(f\left(u_{h}\right), \eta_{0}\left(u_{h}\right), q_{0}\left(u_{h}\right)\right)\right\|_{L_{l o c}^{4 m /(2 m-1)}} \leq C, \quad C \text { independent of } h,
$$

where $\left(\eta_{0}, q_{0}\right)$ is the entropy pair given by (2.2).

3.2. Entropy inequality. We now estimate the entropy inequalities as stated in the third condition in Theorem 2.1 for the entropy functions of form

$$
\eta^{(k)}(u) \sim O\left(|u|^{2 \ell-k}\right), \quad \text { for }|u| \gg 1, k=0,1, \cdots, 2 \ell,
$$

where $\ell \leq 2 m$ is a positive integer. In the next subsection, we will use the integer $\ell \leq(13 m-3) / 6$ in the compactness arguments. We will always take $\ell \leq(3 m+1) / 2$ for using Theorem 2.1 to ensure the entropy inequalities. For any $\phi(x, t) \in C_{0}^{1}$, $\phi \geq 0$, denote $\phi_{j}^{n}=\phi\left(x_{j}, t_{n}\right)$ and

$$
I_{h}(\phi) \equiv h \sum_{j, n} \phi_{j}^{n}\left(\eta\left(u_{j}^{n+1}\right)-\eta\left(u_{j}^{n}\right)\right)+\frac{1}{2} \lambda h \sum_{j, n} \phi_{j}^{n}\left(q\left(u_{j+1}^{n}\right)-q\left(u_{j-1}^{n}\right)\right) .
$$

We decompose $I_{h}$ into the following three terms that are similar to (3.8).

$$
\begin{aligned}
I_{h}(\phi)= & h \sum_{j, n} \phi_{j}^{n} \eta_{j}^{\prime} J_{j}+h \sum_{j, n} \phi_{j}^{n} \eta_{j}^{\prime} H_{j}+h \sum_{j, n} \phi_{j}^{n}\left(\eta_{j}^{\prime} F_{j}+\frac{1}{2} \lambda \Delta_{0} q_{j}\right) \\
& +\frac{1}{2} h \sum_{j, n} \phi_{j}^{n} \eta_{j}^{\prime \prime}\left(F_{j}^{2}+H_{j}^{2}+2 F_{j} H_{j}+J_{j}^{2}+2 J_{j}\left(F_{j}+H_{j}\right)\right) \\
& +h \sum_{k=3}^{2 \ell-1} \frac{1}{k !} \sum_{j, n} \phi_{j}^{n} \eta_{j}^{(k)}\left(F_{j}+H_{j}+J_{j}\right)^{k} \\
& +h \frac{1}{(2 \ell) !} \sum_{j, n} \phi_{j}^{n} \eta^{(2 \ell)}\left(\xi^{*}\right)\left(F_{j}+H_{j}+J_{j}\right)^{2 \ell} \\
= & I_{1}^{h}(\phi)+I_{2}^{h}(\phi)+I_{3}^{h}(\phi) .
\end{aligned}
$$

Notice that there is a remainder term in $I_{3}$ with $\xi^{*} \in\left[u_{j}^{n}, u_{j}^{n+1}\right]$. This term can be estimated by using the fact that $\eta^{(2 \ell)}$ is uniformly bounded for the entropy functions of form (3.32). We now estimate all these terms in the same order as those in Subsection 3.1 to keep consistency. We will skip some parts of the estimates, which are similar to the corresponding parts in the previous subsection.

Estimate of $I_{1}^{h}$. Similar to the estimate (3.10), one can use the identity

$$
\Delta_{+}\left(\phi_{j}^{n} \eta_{j}^{\prime}\right)=\frac{1}{2}\left(\phi_{j}^{n}+\phi_{j+1}^{n}\right) \Delta_{+} \eta_{j}^{\prime}+\frac{1}{2}\left(\eta_{j}^{\prime}+\eta_{j+1}^{\prime}\right) \Delta_{+} \phi_{j}^{n}
$$


and the expression of $J_{j}$ in (3.3) to get

$$
\begin{aligned}
\sum_{j, n} \phi_{j}^{n} \eta_{j}^{\prime} J_{j}= & -\frac{1}{2} \lambda \sum_{j, n} \tilde{\beta}_{j+1 / 2}^{n}\left(\phi_{j}^{n}+\phi_{j+1}^{n}\right) \Delta_{+} \eta_{j}^{\prime}\left|\Delta_{+} a_{j}\right| \Delta_{+} u_{j} \\
& -\frac{1}{2} \lambda \sum_{j, n} \tilde{\beta}_{j+1 / 2}^{n}\left(\eta_{j}^{\prime}+\eta_{j+1}^{\prime}\right) \Delta_{+} \phi_{j}^{n}\left|\Delta_{+} a_{j}\right| \Delta_{+} u_{j} .
\end{aligned}
$$

Applying Lemma 3.1 to the first term on the right-hand side and estimating the second term directly, one has

$$
\begin{aligned}
\sum_{j, n} \phi_{j}^{n} \eta_{j}^{\prime} J_{j} \leq & -\frac{1}{2} \lambda \beta_{0} \sum_{j, n}\left(\phi_{j}^{n}+\phi_{j+1}^{n}\right) \Phi\left(s_{j}\right)\left|\Delta_{+} u_{j}\right|^{3} \\
& +\lambda \beta_{1} C \sum_{j, n} s_{j} \Phi\left(s_{j}\right)\left|\Delta_{+} u_{j}\right|^{2}\left|\Delta_{+} \phi_{j}^{n}\right|
\end{aligned}
$$

where $s_{j}$ and $\Phi(s)$ are determined by (3.11). With this good term in our hands, we now estimate other terms. Notice that

$$
\sum_{j, n} \phi_{j}^{n} \eta_{j}^{\prime} H_{j}=-\frac{1}{2} \lambda^{2} \sum_{j, n} \phi_{j}^{n} \Delta_{+} \eta_{j}^{\prime} \frac{\left(\Delta_{+} f_{j}\right)^{2}}{\Delta_{+} u_{j}}-\frac{1}{2} \lambda^{2} \sum_{j, n} \eta_{j+1}^{\prime} \Delta_{+} \phi_{j}^{n} \frac{\left(\Delta_{+} f_{j}\right)^{2}}{\Delta_{+} u_{j}} .
$$

As in estimating (3.13)-(3.14), expanding $\Delta_{+} \eta_{j}^{\prime}$ and $\Delta_{+} f_{j}$ in the first term on the right-hand side of the above equality and estimating the resulting terms directly, we have

$$
\begin{aligned}
\sum_{j, n} \phi_{j}^{n} \eta_{j}^{\prime} H_{j} \leq & -\frac{1}{2} \lambda^{2} \sum_{j, n} \phi_{j}^{n} \eta_{j}^{\prime \prime}\left(\Delta_{+} f_{j}\right)^{2}+\lambda \varepsilon_{0} C \sum_{j, n} \phi_{j}^{n} \Phi\left(s_{j}\right)\left|\Delta_{+} u_{j}\right|^{3} \\
& +\lambda \varepsilon_{0} C \sum_{j, n} s_{j} \Phi\left(s_{j}\right)\left|\Delta_{+} u_{j}\right|^{2}\left|\Delta_{+} \phi_{j}^{n}\right| .
\end{aligned}
$$

Using Lemma 3.4, one has

$$
\begin{aligned}
& \sum_{j, n} \phi_{j}^{n}\left(\eta_{j}^{\prime} F_{j}+\frac{1}{2} \lambda \Delta_{0} q_{j}\right)=-\frac{1}{2} \lambda \sum_{j, n} \phi_{j}^{n}\left(\eta_{j}^{\prime} \Delta_{0} f_{j}-\Delta_{0} q_{j}\right) \\
& =\frac{1}{4} \lambda \sum_{j, n} \phi_{j}^{n}\left(\eta_{j}^{\prime \prime}\left(\Delta_{+} u_{j}\right)^{2} \Delta_{+} a_{j}-\eta_{j}^{\prime \prime} \int_{u_{j-1}}^{u_{j+1}}\left(u_{j}-s\right)^{2} a^{\prime}(s) d s\right) \\
& \quad-\frac{1}{4} \lambda \sum_{j, n} \phi_{j}^{n} a_{j} \Delta_{+} \eta_{j}^{\prime \prime}\left(\Delta_{+} u_{j}\right)^{2}-\frac{1}{4} \lambda \sum_{j, n} \eta_{j+1}^{\prime \prime} a_{j}\left(\Delta_{+} u_{j}\right)^{2} \Delta_{+} \phi_{j}^{n} \\
& \quad-\frac{1}{4} \lambda \sum_{j, n} \phi_{j}^{n}\left[\eta^{\prime \prime \prime}\left(\xi_{j}\right) \int_{u_{j}}^{u_{j+1}}\left(u_{j}-s\right)^{2} a(s) d s+\eta^{\prime \prime \prime}\left(\xi_{j-1}\right) \int_{u_{j-1}}^{u_{j}}\left(u_{j}-s\right)^{2} a(s) d s\right] .
\end{aligned}
$$

Each term on the right-hand side of the above equality is the sum of products of three differences and, therefore, can be directly estimated by similar arguments as in (3.15)-(3.16).

$$
\begin{aligned}
& \sum_{j, n} \phi_{j}^{n}\left(\eta_{j}^{\prime} F_{j}+\frac{1}{2} \lambda \Delta_{0} q_{j}\right) \\
& \quad \leq \lambda C \sum_{j, n}\left[\left(\phi_{j}^{n}+\phi_{j+1}^{n}\right) \Phi\left(s_{j}\right)\left|\Delta_{+} u_{j}\right|^{3}+s_{j} \Phi\left(s_{j}\right)\left|\Delta_{+} u_{j}\right|^{2}\left|\Delta_{+} \phi_{j}^{n}\right|\right] .
\end{aligned}
$$


Using (3.35)-(3.37), we have

$$
\begin{gathered}
I_{1}^{h}(\phi) \leq-\frac{1}{2} \lambda \beta_{0} h \sum_{j, n}\left(\phi_{j}^{n}+\phi_{j+1}^{n}\right) \Phi\left(s_{j}\right)\left|\Delta_{+} u_{j}\right|^{3}-\frac{1}{2} \lambda^{2} h \sum_{j, n} \phi_{j}^{n} \eta_{j}^{\prime \prime}\left(\Delta_{+} f_{j}\right)^{2} \\
+\lambda\left(1+\varepsilon_{0}+\beta_{1}\right) C h \sum_{j, n}\left[\left(\phi_{j}^{n}+\phi_{j+1}^{n}\right) \Phi\left(s_{j}\right)\left|\Delta_{+} u_{j}\right|^{3}\right. \\
\left.+s_{j} \Phi\left(s_{j}\right)\left|\Delta_{+} u_{j}\right|^{2}\left|\Delta_{+} \phi_{j}^{n}\right|\right] .
\end{gathered}
$$

Estimate of $I_{2}^{h}$. Using the identity (3.19), one can decompose the first term in $I_{2}^{h}$ and estimate similar to that of (3.20) to obtain

$$
\begin{aligned}
\sum_{j, n} \phi_{j}^{n} \eta_{j}^{\prime \prime} F_{j}^{2}= & \frac{1}{2} \lambda^{2} \sum_{j, n} \phi_{j}^{n}\left(\eta_{j}^{\prime \prime}+\eta_{j+1}^{\prime \prime}\right)\left(\Delta_{+} f_{j}\right)^{2}-\frac{1}{4} \lambda^{2} \sum_{j, n} \phi_{j}^{n} \eta_{j}^{\prime \prime}\left(\Delta_{-} \Delta_{+} f_{j}\right)^{2} \\
& +\frac{1}{2} \lambda^{2} \sum_{j, n} \eta_{j+1}^{\prime \prime}\left(\Delta_{+} f_{j}\right)^{2} \Delta_{+} \phi_{j}^{n} \\
\leq & \lambda^{2} \sum_{j, n} \phi_{j}^{n} a_{j+1 / 2}^{2} \eta_{j}^{\prime \prime}\left(\Delta_{+} u_{j}\right)^{2}-\frac{1}{4} \lambda^{2} \sum_{j, n} \phi_{j}^{n} \eta_{j}^{\prime \prime}\left(\Delta_{-} \Delta_{+} f_{j}\right)^{2} \\
& +\lambda \varepsilon_{0} C \sum_{j, n}\left[\left(\phi_{j}^{n}+\phi_{j+1}^{n}\right) \Phi\left(s_{j}\right)\left|\Delta_{+} a_{j}\right|^{3}+s_{j} \Phi\left(s_{j}\right)\left|\Delta_{+} u_{j}\right|^{2}\left|\Delta_{+} \phi_{j}^{n}\right|\right] .
\end{aligned}
$$

Estimating the second term of $I_{2}^{h}$ is similar to that of (3.21)-(3.22) by

$$
\begin{aligned}
\sum_{j, n} \phi_{j}^{n} \eta_{j}^{\prime \prime} H_{j}^{2} \leq & \frac{1}{2} \lambda^{4} \sum_{j, n} \phi_{j}^{n} \eta_{j}^{\prime \prime}\left(\Delta_{-} \Delta_{+} f_{j}\right)^{2}\left(\frac{\Delta_{+} f_{j-1}}{\Delta_{+} u_{j-1}}\right)^{2} \\
& +\lambda \varepsilon_{0}^{3} C \sum_{j, n}\left(\phi_{j}^{n}+\phi_{j+1}^{n}\right) \Phi\left(s_{j}\right)\left|\Delta_{+} u_{j}\right|^{3}
\end{aligned}
$$

The main idea in estimating the third term in $I_{2}^{h}$ is to utilize the symmetric property. It involves some similar techniques as in estimating (3.23) and (3.24). We omit detailed calculations here and write down the resulting terms as follows:

$$
\begin{aligned}
& \sum_{j, n} \phi_{j}^{n} \eta_{j}^{\prime \prime} F_{j} H_{j}=-\frac{1}{4} \lambda^{3} \sum_{j, n} \phi_{j}^{n} \eta_{j}^{\prime \prime} \Delta_{0} f_{j} \Delta_{-}\left(\frac{\left(\Delta_{+} f_{j}\right)^{2}}{\Delta_{+} u_{j}}\right) \\
&=-\frac{1}{8} \lambda^{3} \sum_{j, n}\left[\left(\sqrt{\eta_{j}^{\prime \prime}} \Delta_{+} f_{j}\right)^{2} \Delta_{0}\left(\phi_{j}^{n} \frac{\Delta_{+} f_{j}}{\Delta_{+} u_{j}}\right)\right.\left.-\Delta_{+}\left(\phi_{j}^{n} \frac{\Delta_{+} f_{j}}{\Delta_{+} u_{j}}\right)\left(\Delta_{+}\left(\sqrt{\eta_{j}^{\prime \prime}} \Delta_{+} f_{j}\right)\right)^{2}\right] \\
&+\frac{1}{4} \lambda^{3} \sum_{j, n} \sqrt{\eta_{j}^{\prime \prime}}\left[\frac{\left(\Delta_{+} f_{j}\right)^{2}}{\Delta_{+} u_{j}} \Delta_{-}\left(\Delta_{+}\left(\phi_{j}^{n} \sqrt{\eta_{j}^{\prime \prime}}\right) \Delta_{+} f_{j}\right)\right. \\
&\left.+\phi_{j}^{n} \Delta_{0} f_{j} \frac{\left(\Delta_{+} f_{j-1}\right)^{2}}{\Delta_{+} u_{j-1}} \Delta_{-} \sqrt{\eta_{j}^{\prime \prime}}\right] .
\end{aligned}
$$


Clearly the terms on the right-hand side of the above equality are the sums of three difference products of $u_{j}^{n}$ and hence can be estimated directly.

$$
\begin{aligned}
\sum_{j, n} \phi_{j}^{n} \eta_{j}^{\prime \prime} F_{j} H_{j} \leq & \lambda \varepsilon_{0}^{2} C \sum_{j, n}\left(\phi_{j}^{n}+\phi_{j+1}^{n}\right) \Phi\left(s_{j}\right)\left|\Delta_{+} u_{j}\right|^{3} \\
& +\lambda \varepsilon_{0}^{2} C \sum_{j, n} s_{j} \Phi\left(s_{j}\right)\left|\Delta_{+} u_{j}\right|^{2}\left(\left|\Delta_{+} \phi_{j}^{n}\right|+\left|\Delta_{-} \phi_{j}^{n}\right|\right) .
\end{aligned}
$$

The remainder three terms in $I_{2}^{h}$ can be similarly estimated as in the estimates (3.26) and (3.27).

$$
\begin{aligned}
& \sum_{j, n} \phi_{j}^{n} \eta_{j}^{\prime \prime} J_{j}^{2}+2 \sum_{j, n} \phi_{j}^{n} \eta_{j}^{\prime \prime} J_{j}\left(F_{j}+H_{j}\right) \\
& \quad \leq \lambda \varepsilon_{0}\left(\beta_{1}+\beta_{1}^{2}\right) C \sum_{j, n}\left(\phi_{j}^{n}+\phi_{j+1}^{n}\right) \Phi\left(s_{j}\right)\left|\Delta_{+} u_{j}\right|^{3} .
\end{aligned}
$$

Therefore, combining (3.39)-(3.40) with (3.41)-(3.42), we have the following estimate for $I_{2}^{h}$ :

$$
\begin{aligned}
I_{2}^{h}(\phi) \leq & \frac{1}{2} \lambda^{2} h \sum_{j, n} \phi_{j}^{n} \eta_{j}^{\prime \prime}\left(\Delta_{+} f_{j}\right)^{2}-\frac{1}{8} \lambda^{2} h \sum_{j, n} \phi_{j}^{n}\left(1-2 \varepsilon_{0}^{2}\right) \eta_{j}^{\prime \prime}\left(\Delta_{-} \Delta_{+} f_{j}\right)^{2} \\
& +\lambda C \varepsilon_{0}\left(1+\varepsilon_{0}+\varepsilon_{0}^{2}+\beta+\beta^{2}\right) h \sum_{j, n}\left(\phi_{j}^{n}+\phi_{j+1}^{n}\right) \Phi\left(s_{j}\right)\left|\Delta_{+} u_{j}\right|^{3} \\
& +\lambda \varepsilon_{0}\left(1+\varepsilon_{0}\right) C h \sum_{j, n} s_{j} \Phi\left(s_{j}\right)\left|\Delta_{+} u_{j}\right|^{2}\left(\left|\Delta_{+} \phi_{j}^{n}\right|+\left|\Delta_{-} \phi_{j}^{n}\right|\right) .
\end{aligned}
$$

Estimating $I_{3}^{h}$ is straightforward as in that of (3.29) and (3.30). One can obtain

$$
I_{3}^{h}(\phi) \leq \lambda C h \sum_{k=3}^{4 m}\left(\varepsilon_{0}^{k-1}+\varepsilon_{0}^{2 k-1}+\varepsilon_{0}^{k-1} \beta^{k}\right) \sum_{j, n}\left(\phi_{j}^{n}+\phi_{j+1}^{n}\right) \Phi\left(s_{j}\right)\left|\Delta_{+} u_{j}\right|^{3} .
$$

Finally, we have the following estimate of $I_{h}$ after combining (3.38) with (3.43)(3.44):

$$
\begin{aligned}
I_{h}(\phi) \leq & -\frac{1}{4} \lambda \beta_{0} h \sum_{j, n}\left(\phi_{j}^{n}+\phi_{j+1}^{n}\right) \Phi\left(s_{j}\right)\left|\Delta_{+} u_{j}\right|^{3} \\
& -\frac{1}{8} \lambda^{2}\left(1-2 \varepsilon_{0}^{2}\right) h \sum_{j, n} \phi_{j}^{n} \eta_{j}^{\prime \prime}\left(\Delta_{-} \Delta_{+} f_{j}\right)^{2} \\
& +\lambda\left(1+\varepsilon_{0}+\beta_{1}\right) C h \sum_{j, n} s_{j} \Phi\left(s_{j}\right)\left|\Delta_{+} u_{j}\right|^{2}\left(\left|\Delta_{+} \phi_{j}^{n}\right|+\left|\Delta_{-} \phi_{j}^{n}\right|\right) \\
& +\lambda C\left(1+\varepsilon_{0} \beta_{1}+\sum_{k=2}^{4 m} \varepsilon_{0}^{k-1} \beta_{1}^{k}\right) h \sum_{j, n}\left(\phi_{j}^{n}+\phi_{j+1}^{n}\right) \Phi\left(s_{j}\right)\left|\Delta_{+} u_{j}\right|^{3} .
\end{aligned}
$$

Choose $\beta_{0}$ large and $\varepsilon_{0} \beta_{1}$ small. Then we have

$$
\begin{aligned}
I_{h}(\phi) \leq & -\frac{1}{8} \lambda \beta_{0} h \sum_{j, n}\left(\phi_{j}^{n}+\phi_{j+1}^{n}\right) \Phi\left(s_{j}\right)\left|\Delta_{+} u_{j}\right|^{3} \\
& +\lambda \beta_{1} C h \sum_{j, n} s_{j} \Phi\left(s_{j}\right)\left|\Delta_{+} u_{j}\right|^{2}\left(\left|\Delta_{+} \phi_{j}^{n}\right|+\left|\Delta_{-} \phi_{j}^{n}\right|\right) .
\end{aligned}
$$


Now we deal with the last term in the above inequality. From the Hölder inequality, one has

$$
\begin{gathered}
\lambda h \sum_{j, n} s_{j} \Phi\left(s_{j}\right)\left|\Delta_{+} u_{j}\right|^{2}\left(\left|\Delta_{+} \phi_{j}^{n}\right|+\left|\Delta_{-} \phi_{j}^{n}\right|\right) \leq \lambda h^{1+\alpha}\|\phi\|_{C^{\alpha}} \sum_{\left(x_{j}, t_{n}\right) \in \Omega} \Phi\left(s_{j}\right) s_{j}\left(\Delta_{+} u\right)^{2} \\
\quad \leq C h^{\alpha-1 / 3}\|\phi\|_{C^{\alpha}}\left(\sum_{\left(x_{j}, t_{n}\right) \in \Omega} s_{j}^{6 \ell-9 m+3} \tau_{n} h\right)^{1 / 3}\left(\sum_{\left(x_{j}, t_{n}\right) \in \Omega} \Phi\left(s_{j}\right)\left|\Delta_{+} u\right|^{3} h\right)^{2 / 3} .
\end{gathered}
$$

Let $6 \ell-9 m+3 \leq 4 m$. Then

$$
\lambda h \sum_{j, n} s_{j} \Phi\left(s_{j}\right)\left|\Delta_{+} u_{j}\right|^{2}\left(\left|\Delta_{+} \phi_{j}^{n}\right|+\left|\Delta_{-} \phi_{j}^{n}\right|\right) \leq C h^{\alpha-1 / 3}\|\phi\|_{C^{\alpha}} .
$$

Substituting it back to (3.33), we have the following proposition.

Proposition 3.2. Under the same assumptions of Theorem 3.1,

$$
\sum_{j, n} \phi_{j}^{n}\left(\eta\left(u_{j}^{n+1}\right)-\eta\left(u_{j}^{n}\right)\right) h+\frac{1}{2} \lambda \sum_{j, n} \phi_{j}^{n}\left(q\left(u_{j+1}^{n}\right)-q\left(u_{j-1}^{n}\right)\right) h \leq C h^{\alpha-1 / 3}\|\phi\|_{C^{\alpha}}
$$

holds for any $\phi \in C_{0}^{1}, \phi \geq 0$, where $(\eta, q)$ is the entropy pair given by (3.32).

3.3. $H^{-1}$ compactness of entropy dissipation measures. For the entropy pair $(\eta, q)$, we define the following functional as the entropy dissipation measures

$$
M_{h}(\phi)=\iint\left(\eta\left(u_{h}\right) \partial_{t} \phi+q\left(u_{h}\right) \partial_{x} \phi\right) d x d t, \quad \phi \in C_{0}^{1},
$$

for the approximate solutions $u_{h}(x, t)$ defined by (3.4). In this subsection, we will show that $M_{h}$ is compact in $H^{-1}$ for the entropy pairs $(u, f(u))$ and $\left(\eta_{0}, q_{0}\right)$ with $\eta_{0}(u)=u^{2}$.

From Proposition 3.1, we can easily get from the Hölder inequality that $\left|M_{h}(\phi)\right|$

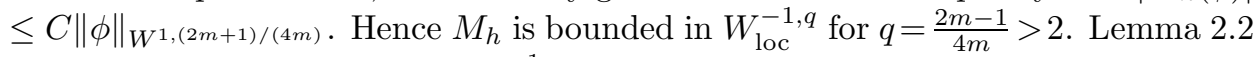
tells us that $M_{h}$ is compact in $H_{\text {loc }}^{-1}$ as long as we can prove that $M_{h}$ is compact in $W_{\text {loc }}^{-1, p}$ for some $p<2$. We define the following two functionals:

$$
I_{h}(\phi)=\sum_{j, n} \phi_{j}^{n}\left(\eta\left(u_{j}^{n+1}\right)-\eta\left(u_{j}^{n}\right)\right) h+\frac{1}{2} \lambda \sum_{j, n} \phi_{j}^{n}\left(q\left(u_{j+1}^{n}\right)-q\left(u_{j-1}^{n}\right)\right) h,
$$

and $\Delta_{h}(\phi)=I_{h}(\phi)+M_{h}(\phi)$ for any strictly convex entropy $(\eta, q)$ and $\phi \in C_{0}^{1}$. It is easy to show that

$$
\begin{aligned}
\Delta_{h}(\phi)= & -\sum_{j, n}\left(\eta_{j}^{n+1}-\eta_{j}^{n}\right) \int_{x_{j-1 / 2}}^{x_{j+1 / 2}}\left(\phi\left(x, t_{n+1}\right)-\phi\left(x_{j}, t_{n}\right)\right) d x \\
& -\frac{1}{2} \sum_{j, n}\left(q_{j+1}^{n}-q_{j}^{n}\right) \int_{t_{n}}^{t_{n+1}}\left(2 \phi\left(x_{j+1 / 2}, t\right)-\phi\left(x_{j}, t_{n}\right)-\phi\left(x_{j+1}, t_{n}\right)\right) d t .
\end{aligned}
$$


Estimating directly and using the Hölder inequality and Proposition 3.1, one obtains

$$
\begin{aligned}
& \left|\Delta_{h}(\phi)\right| \leq\|\phi\|_{C^{\alpha}} \sum_{\left(x_{j}, t_{n}\right) \in \Omega}\left(\left|\eta_{j}^{n+1}-\eta_{j}^{n}\right| h^{1+\alpha}+\left|q_{j+1}^{n}-q_{j}^{n}\right| \tau_{n} h^{\alpha}\right) \\
& \quad \leq h^{1+\alpha}\|\phi\|_{C^{\alpha}} \sum_{\left(x_{j}, t_{n}\right) \in \Omega}\left(\sum_{k=1}^{2} \frac{3^{k}}{k !}\left|\eta_{j}^{(k)}\right|\left(\left|F_{j}\right|^{k}+\left|H_{j}\right|^{k}+\left|J_{j}\right|^{k}\right)+\lambda\left|q^{\prime}\left(\xi_{j}\right)\right|\left|\Delta_{+} u_{j}^{n}\right|\right) \\
& \quad \leq \lambda C h^{1+\alpha}\|\phi\|_{C^{\alpha}} \sum_{\left(x_{j}, t_{n}\right) \in \Omega} \Phi\left(s_{j}\right)^{1 / 3}\left|\Delta_{+} u_{j}^{n}\right| \\
& \quad \leq \lambda^{1 / 3} C h^{\alpha-2 / 3}\|\phi\|_{C^{\alpha}} \rightarrow 0, \quad \text { for } \frac{2}{3}<\alpha<1 .
\end{aligned}
$$

This implies that $\Delta_{h}$ is compact in $W_{\text {loc }}^{-1, p_{1}}$ for $p_{1}<\frac{2}{1+\alpha}<2$. We know from Proposition 3.2 that $I_{h}$ can be split as $I_{h}(\phi)=I_{1}^{h}(\phi)+I_{2}^{h}(\phi)$ such that

$$
I_{1}^{h} \leq 0, \quad\left|I_{2}^{h}(\phi)\right| \leq C h^{\alpha-1 / 3}\|\phi\|_{C^{\alpha}} .
$$

Consequently, we have that $I_{2}^{h}$ is compact in $W_{\text {loc }}^{-1, p_{2}}$ for $1<p_{2}<2$. The remainder is to show that $I_{1}^{h}$ is also compact in $W_{\mathrm{loc}}^{-1, p}$ for some $p<2$. Since $I_{1}^{h}$ is negative due to (3.50), we know from Lemma 2.1 that we only need to show that $I_{1}^{h}$ is bounded in $W_{\mathrm{loc}}^{-1, p}$ for some $p<2$. This is a direct consequence of (3.46), (3.49), and (3.50). Thus, we have the following proposition.

Proposition 3.3. Under the same assumptions of Theorem 3.1,

$$
\partial_{t} u_{h}+\partial_{x} f\left(u_{h}\right), \quad \partial_{t} \eta_{0}\left(u_{h}\right)+\partial_{x} q_{0}\left(u_{h}\right) \quad \text { are compact in } H_{\text {loc }}^{-1}
$$

where $\left(\eta_{0}, q_{0}\right)$ is the entropy pair given by $(2.2)$.

As a consequence of Theorem 2.1, Corollary 3.1, Proposition 3.2, and Proposition 3.3, we conclude Theorem 3.1 for the convex scalar conservation laws.

\section{Extension to The NONCONVEX CASE}

For the nonconvex scalar conservation laws, the second-order numerical viscosity term

$$
\lambda \Delta_{-}\left(\beta_{j+1 / 2}^{n}\left|\Delta_{+} f^{\prime}\left(u_{j}^{n}\right)\right| \Delta_{+} u_{j}^{n}\right)
$$

is degenerate at the points $\left\{u \mid f^{\prime \prime}(u)=0\right\}$. Typically such a degenerate point occurs near the contact discontinuities, which is quite sensitive to the stability of the discontinuities.

To assume the stability, we consider the following second-order numerical viscosity in the Lax-Wendroff schemes:

$$
\lambda \Delta_{-}\left(\beta_{j+1 / 2}^{n}\left|\Delta_{+} u_{j}^{n}\right| \Delta_{+} u_{j}^{n}\right)
$$

with $\beta_{j+1 / 2}^{n} \in\left(\beta_{0}+O\left(\left|a^{\prime}\right|\right), \beta_{1}+O\left(\left|a^{\prime}\right|\right)\right), \beta_{0}>0$, for the nonconvex scalar case.

This covers both the convex case (the convexity implies $\beta_{0}>0$ ) and the nonconvex case. With such a numerical viscosity we can use the same arguments as in Section 3 , when $C \gg 1$ and $\epsilon_{0} \ll 1$, to obtain the same viscosity estimate

$$
\sum_{j, n} \Phi\left(s_{j}\right)\left|u_{j}^{n}-u_{j+1}^{n}\right|^{3} h \leq C
$$


and entropy estimate

$$
\sum_{j} \eta\left(u_{j}^{n+1}\right) \leq \sum_{j} \eta\left(u_{j}^{n}\right)
$$

for $\eta^{\prime \prime}=u^{4 m}$. Using these estimates, we can similarly verify that the corresponding approximate solutions $u_{h}(x, t)$ satisfy the three conditions in Theorem 2.1 for any convex entropy pair. Therefore we have the following convergence theorem for the scalar conservation laws (1.1) whose flux functions satisfy

$$
\text { meas }\left\{u \mid f^{\prime \prime}(u)=0\right\}=0, \quad f^{(k)}(u) \sim O\left(|u|^{m-k}\right), \quad \text { for }|u| \gg 1,0 \leq k \leq m .
$$

Theorem 4.1. Let $u_{h}(x, t)$ be numerical approximate solutions, generated from the Lax-Wendroff scheme (1.2) endowed with the numerical viscosity (4.1), of the scalar conservation laws (1.1) satisfying (4.4). Assume that the coefficient $\beta_{0}$ in (4.1) is suitably large and the CFL number $\varepsilon_{0}$ given by (1.4) is suitably small. Then the sequence $u_{h}$ has a strong convergent subsequence and the limit function is the entropy solution of (1.1) satisfying the entropy inequalities $\partial_{t} \eta(u)+\partial_{x} q(u) \leq 0$ in $\mathcal{D}^{\prime}$, for any convex entropy pair $(\eta, q)$ satisfying $\eta^{(k)}(u) \sim O\left(|u|^{r-k}\right)$, for $|u| \gg 1$, $0 \leq k \leq r<4 m$.

Proof. Only one thing we should check is that, for any such a $C^{2}$ entropy pair $(\eta(u), q(u)), \partial_{t} \eta\left(u_{h}\right)+\partial_{x} q\left(u_{h}\right)$ is compact in $H_{\text {loc }}^{-1}$. This can be achieved as follows. For any $C^{2}$ entropy pair $(\eta, q)$, there exists $C_{0}>0$ such that $\left|\eta^{\prime \prime}\right| \leq C_{0}\left|\eta_{*}^{\prime \prime}\right|$ where $\eta_{*}$ is a strictly convex entropy. Define $\bar{\eta}=C_{0} \eta_{*}-\eta$. Then $\bar{\eta}$ is a convex entropy. Noting that both $\partial_{t} \bar{\eta}\left(u_{h}\right)+\partial_{x} \bar{q}\left(u_{h}\right)$ and $\partial_{t} \eta_{*}\left(u_{h}\right)+\partial_{x} q_{*}\left(u_{h}\right)$ are compact sets in $H_{\text {loc }}^{-1}$, one obtains that $\partial_{t} \eta\left(u_{h}\right)+\partial_{x} q\left(u_{h}\right)$ is compact in $H_{\text {loc }}^{-1}$. This completes the proof.

Remark. The assumption (4.4) can be relaxed to more general flux functions for the Lax-Wendroff scheme. The strong convergence becomes weak one in $L^{r}, r<4 m$, and the limit function is the weak solution satisfying the entropy condition for the Young measures $\nu_{x, t}$ determined by the Lax-Wendroff approximate solutions $u_{h}(x, t)$ :

$$
\partial_{t}\left\langle\nu_{x, t}, \eta\right\rangle+\partial_{x}\left\langle\nu_{x, t}, q\right\rangle \leq 0
$$

for any convex entropy pair $(\eta, q)$ in the above theorem.

\section{EXtension to hyperbolic Systems}

We write the Lax-Wendroff scheme in the following form

$$
u_{j}^{n+1}=u_{j}^{n}+F_{j}^{n}+H_{j}^{n}+J_{j}^{n} .
$$

Here

$$
\begin{gathered}
F_{j}^{n}=-\frac{1}{2} \lambda \Delta_{0} f\left(u_{j}^{n}\right), \quad H_{j}^{n}=\frac{1}{2} \lambda^{2} \Delta_{-}\left(a\left(\frac{u_{j}^{n}+u_{j+1}^{n}}{2}\right) \Delta_{+} f\left(u_{j}^{n}\right)\right), \\
J_{j}^{n}=\lambda \Delta_{-}\left(\beta_{j+1 / 2}^{n}\left|\Delta_{+} u_{j}^{n}\right| \Delta_{+} u_{j}^{n}\right),
\end{gathered}
$$

where the scalar function $\beta_{j+1 / 2}^{n} \geq \beta_{0}>0$, for some $\beta_{0}$, is smooth with respect to $u_{j}^{n}$ and $u_{j+1}^{n}$ and is bounded from below. We assume that

$$
\sup _{j, n}\left|u_{j}^{n}\right| \leq M .
$$


In this section we show the convergence of such a scheme for $2 \times 2$ systems and the entropy consistency of the boundedly convergent Lax-Wendroff approximate solutions with general hyperbolic systems of conservation laws with a convex entropy. This depends on the following estimates.

5.1. Dissipation estimates. We first start with the entropy estimate. Let $\eta$ be a $C^{2}$ convex entropy function. As in the scalar case, we take the Taylor expansion to the time increment of the entropy to get

$$
\begin{aligned}
\sum_{j}\left(\eta\left(u_{j}^{n+1}\right)-\eta\left(u_{j}^{n}\right)\right)= & \sum_{j} \eta^{\prime}\left(u_{j}^{n}\right)\left(u_{j}^{n+1}-u_{j}^{n}\right) \\
& +\frac{1}{2} \sum_{j}\left(u_{j}^{n+1}-u_{j}^{n}\right)^{\top} \eta^{\prime \prime}\left(u_{j}^{n}\right)\left(u_{j}^{n+1}-u_{j}^{n}\right) \\
& +\sum_{j} O\left(\left|u_{j}^{n+1}-u_{j}^{n}\right|^{3}\right) \equiv I_{1}+I_{2}+I_{3} .
\end{aligned}
$$

We will keep the same order of the estimates as in Section 3 and will only emphasize the new features, which are different from the scalar case. The arguments similar to those in Section 3 will be omitted. We point out here that some of the estimates in this section are simpler than the ones in Section 3 since we assumed the uniform boundedness of the approximate solutions.

Estimate of $I_{1} . \quad$ As in (3.11), we directly estimate that

$$
\sum_{j}\left(\eta_{j}^{\prime}\right)^{\top} J_{j}=-\lambda \sum_{j} \beta_{j+1 / 2}\left|\Delta_{+} u_{j}\right| \Delta_{+}\left(\eta_{j}^{\prime}\right)^{\top} \Delta_{+} u_{j} \leq-\lambda \beta_{0} \sum_{j}\left|\Delta_{+} u_{j}\right|^{3},
$$

using the expression of $J_{j}$ in (5.2), the summation by parts, and the convexity of $\eta$. This gives us the main dissipative term. Here we used the notation $\beta_{j+1 / 2}=$ $\beta\left(u_{j}, u_{j+1}\right)$, which is a scalar function. We remark that (5.5) is the only place we restrict the viscosity $\beta\left(u_{j}, u_{j+1}\right)$ to be a scalar function.

As in estimating (3.13) and (3.14), we have

$$
\begin{aligned}
\sum_{j}\left(\eta_{j}^{\prime}\right)^{\top} H_{j} & =-\frac{1}{2} \lambda^{2} \sum_{j}\left(\Delta_{+} \eta_{j}^{\prime}\right)^{\top} a_{j+1 / 2} \Delta_{+} f_{j} \\
& =-\frac{1}{2} \lambda^{2} \sum_{j}\left(\Delta_{+} u_{j}\right)^{\top} \eta_{j}^{\prime \prime} a_{j} \Delta_{+} f_{j}+\lambda \varepsilon_{0} \sum_{j} O\left(\left|\Delta_{+} u_{j}\right|^{3}\right) .
\end{aligned}
$$

Notice that the necessary and sufficient condition for a function $\eta$ to be an entropy is that $\eta^{\prime \prime} f^{\prime}$ is symmetric, that is, $\eta_{j}^{\prime \prime} a_{j}=\left(a_{j}\right)^{\top} \eta_{j}^{\prime \prime}$. One has from (5.6)

$$
\sum_{j}\left(\eta_{j}^{\prime}\right)^{\top} H_{j}=-\frac{1}{2} \lambda^{2} \sum_{j}\left(\Delta_{+} f_{j}\right)^{\top} \eta_{j}^{\prime \prime} \Delta_{+} f_{j}+\lambda \varepsilon_{0} \sum_{j} O\left(\left|\Delta_{+} u_{j}\right|^{3}\right) .
$$

In estimating the first term of $I_{1}$, we use the conservative property of entropy. Denote $\bar{u}_{j}=\frac{1}{2}\left(u_{j-1}+u_{j+1}\right)$. Similar to (3.16)-(3.17), one can obtain

$$
\sum_{j}\left(\eta_{j}^{\prime}\right)^{\top} F_{j}=-\frac{1}{2} \lambda \sum_{j}\left(\eta_{j}^{\prime}\right)^{\top} \int_{-1 / 2}^{1 / 2} a\left(\bar{u}_{j}+\theta \Delta_{0} u_{j}\right) d \theta \Delta_{0} u_{j}=\lambda \varepsilon_{0} \sum_{j} O\left(\left|\Delta_{+} u_{j}\right|^{3}\right) .
$$

Combining (5.5) with (5.7)-(5.8), we have the following estimate of $I_{1}$.

$$
I_{1} \leq-\lambda \beta_{0} \sum_{j}\left|\Delta_{+} u_{j}\right|^{3}+\lambda \varepsilon_{0} C\left|\Delta_{+} u_{j}\right|^{3}-\frac{1}{2} \lambda^{2} \sum_{j}\left(\Delta_{+} f_{j}\right)^{\top} \eta_{j}^{\prime \prime} \Delta_{+} f_{j} .
$$


Estimate of $I_{2}$. As in (3.19), we substitute (5.1)-(5.2) into $I_{2}$ and have the following expansion.

$$
\begin{aligned}
I_{2}= & \frac{1}{2} \sum_{j} F_{j}^{\top} \eta_{j}^{\prime \prime} F_{j}+\frac{1}{2} \sum_{j} H_{j}^{\top} \eta_{j}^{\prime \prime} H_{j}+\sum_{j} F_{j}^{\top} \eta_{j}^{\prime \prime} H_{j} \\
& +\frac{1}{2} \sum_{j} J_{j}^{\top} \eta_{j}^{\prime \prime} J_{j}+\sum_{j} J_{j}^{\top} \eta_{j}^{\prime \prime}\left(F_{j}+H_{j}\right) .
\end{aligned}
$$

The estimate of the first term in (5.10) is similar to that in (3.20)-(3.22) by the identity (3.20). Putting all products of three differences together in $O\left(\left|\Delta_{+} u_{j}\right|^{3}\right)$, one has

$$
\begin{aligned}
\sum_{j} F_{j}^{\top} \eta_{j}^{\prime \prime} F_{j}= & \lambda^{2} \sum_{j}\left(\Delta_{+} f_{j}\right)^{\top} \eta_{j}^{\prime \prime} \Delta_{+} f_{j}-\frac{1}{4} \lambda^{2} \sum_{j}\left(\Delta_{-} \Delta_{+} f_{j}\right)^{\top} \eta_{j}^{\prime \prime} \Delta_{-} \Delta_{+} f_{j} \\
& +\lambda \varepsilon_{0} \sum_{j} O\left(\left|\Delta_{+} u_{j}\right|^{3}\right) .
\end{aligned}
$$

The estimate of the second term in (5.10) is similar to that in (3.23)-(3.24). After putting all products of three differences in $O\left(\left|\Delta_{+} u_{j}^{n}\right|\right)$, the remainder term is the sum of products of two second differences, which can be controlled by the last term in $(5.11)$.

$$
\begin{aligned}
\sum_{j} H_{j}^{\top} \eta_{j}^{\prime \prime} H_{j} & =\frac{1}{4} \lambda^{4} \sum_{j} \Delta_{-}\left(a_{j+1 / 2} \Delta_{+} f_{j}\right)^{\top} \eta_{j}^{\prime \prime} \Delta_{-}\left(a_{j+1 / 2} \Delta_{+} f_{j}\right) \\
& =\frac{1}{4} \lambda^{4} \sum_{j}\left(\Delta_{-} \Delta_{+} f_{j}\right)^{\top} a_{j}^{\top} \eta_{j}^{\prime \prime} a_{j} \Delta_{-} \Delta_{+} f_{j}+\lambda \varepsilon_{0}^{3} \sum_{j} O\left(\left|\Delta_{+} u_{j}\right|^{3}\right) .
\end{aligned}
$$

The main idea in estimating the third term in (5.10) is to utilize the symmetric property so that every term can be combined into the product of three differences. For any symmetric matrix $A$, we have as in Lemma 3.1 that

$$
-\sum_{j}\left(\Delta_{0} b_{j}\right)^{\top} A b_{j}=\frac{1}{2} \sum_{j} b_{j}^{\top} \Delta_{0} A b_{j}-\frac{1}{2} \sum_{j}\left(\Delta_{+} b_{j}\right)^{\top} \Delta_{+} A \Delta_{+} b_{j} .
$$

Similar to (3.25)-(3.27), we have

$$
\begin{aligned}
\sum_{j} F_{j}^{\top} \eta_{j}^{\prime \prime} H_{j} & =-\frac{1}{4} \lambda^{3} \sum_{j}\left(\Delta_{0} f_{j}\right)^{\top} \eta_{j}^{\prime \prime} \Delta_{-}\left(a_{j+1 / 2} \Delta_{+} u_{j}\right) \\
& =\frac{1}{4} \lambda^{3} \sum_{j} \Delta_{0}\left(\sqrt{\eta_{j}^{\prime \prime}} \Delta_{+} f_{j}\right)^{\top} \sqrt{\eta_{j}^{\prime \prime}} a_{j} \Delta_{+} u_{j}+\lambda \varepsilon_{0}^{2} \sum_{j} O\left(\left|\Delta_{+} u_{j}\right|^{3}\right) .
\end{aligned}
$$

We know from (5.6) that $\sqrt{\eta_{j}^{\prime \prime}} a_{j}\left(\sqrt{\eta_{j}^{\prime \prime}}\right)^{-1}$ is symmetric. Hence we can apply the identity (5.13) to the first term on the right-hand side of the above equality with $b_{j}$ replaced by $\sqrt{\eta_{j}^{\prime \prime}} \Delta_{+} f_{j}$. As a result that every term now is decomposed as the product of three differences, we obtain

$$
\sum_{j} F_{j}^{\top} \eta_{j}^{\prime \prime} H_{j}=\lambda \varepsilon_{0}^{2} \sum_{j} O\left(\left|\Delta_{+} u_{j}\right|^{3}\right)
$$

The estimate of the last three terms in (5.10) is rather straightforward since every term is the sum of products of more than three differences. One has from a 
direct estimate that

$$
\sum_{j} J_{j}^{\top} \eta_{j}^{\prime \prime} J_{j}+2 \sum_{j} J_{j}^{\top} \eta_{j}^{\prime \prime}\left(F_{j}+H_{j}\right) \leq \lambda \varepsilon_{0} \sum_{j} O\left(\left|\Delta_{+} u_{j}\right|^{3}\right) .
$$

Finally, combining (5.11)-(5.12) with (5.14)-(5.15), we have

$$
\begin{aligned}
I_{2} \leq & \frac{1}{2} \lambda^{2} \sum_{j}\left(\Delta_{+} f_{j}\right)^{\top} \eta_{j}^{\prime \prime} \Delta_{+} f_{j}-\frac{1}{8} \lambda^{2} \sum_{j}\left(\Delta_{-} \Delta_{+} f_{j}\right)^{\top} \eta_{j}^{\prime \prime} \Delta_{-} \Delta_{+} f_{j} \\
& +\frac{1}{4} \lambda^{4} \sum_{j}\left(\Delta_{-} \Delta_{+} f_{j}\right)^{\top} a_{j}^{\top} \eta_{j}^{\prime \prime} a_{j} \Delta_{-} \Delta_{+} f_{j}+\lambda \varepsilon_{0} \sum_{j} O\left(\left|\Delta_{+} u_{j}\right|^{3}\right) .
\end{aligned}
$$

Estimating $I_{3}$ simply follows from the fact that each term is the sum of products of more than three difference factors

$$
\left|I_{3}\right| \leq C \sum_{j}\left(\left|F_{j}\right|^{3}+\left|H_{j}\right|^{3}+\left|J_{j}\right|^{3}\right) \leq \lambda \varepsilon_{0}^{2} C \sum_{j}\left|\Delta_{+} u_{j}\right|^{3} .
$$

Combining (5.9) with (5.16), we have

$$
\begin{aligned}
\sum_{j}\left(\eta_{j}^{n+1}-\eta_{j}^{n}\right) \leq & -\lambda \beta_{0} \sum_{j}\left|\Delta_{+} u_{j}\right|^{3}-\frac{1}{8} \lambda^{2}\left(1-\varepsilon_{0}^{2}\right) \sum_{j}\left(\Delta_{-} \Delta_{+} f_{j}\right)^{\top} \eta_{j}^{\prime \prime} \Delta_{-} \Delta_{+} f_{j} \\
& +\lambda\left(1+\varepsilon_{0}+\varepsilon_{0}^{2}\right) C \sum_{j}\left|\Delta_{+} u_{j}\right|^{3} .
\end{aligned}
$$

For $\beta_{0}$ large and $\varepsilon_{0}$ small, we have

$$
\sum_{j}\left(\eta_{j}^{n+1}-\eta_{j}^{n}\right) \leq-\lambda C_{1} \sum_{j}\left|\Delta_{+} u_{j}\right|^{3}-\lambda^{2} C_{2} \sum_{j}\left|\Delta_{-} \Delta_{+} f_{j}\right|^{2} .
$$

This gives

Proposition 5.1. Let $u_{j}^{n}$ be the Lax-Wendroff approximate solutions (5.1)-(5.2) of the hyperbolic systems of conservation laws (1.1) with a convex entropy $\eta$. Assume that $u_{h}$ is uniformly bound and the coefficient $\beta$ in (5.2) is suitably large and the CFL number $\varepsilon_{0}$ given by (1.3) is suitably small. Then

$$
\sum_{j} \eta\left(u_{j}^{n}\right) h+\lambda \sum_{k \leq n ; j}\left|\Delta_{+} u_{j}^{k}\right|^{3} h+\lambda^{2} \sum_{k \leq n ; j}\left|\Delta_{-} \Delta_{+} f\left(u_{j}^{k}\right)\right|^{2} h \leq C .
$$

5.2. $H^{-1}$ compactness estimates. For any $C^{2}$ convex entropy pair $(\eta, q)$ and any $\phi(x, t) \in C_{0}^{1}, \phi \geq 0$, we denote $\phi_{j}^{n}=\phi\left(x_{j}, t_{n}\right)$ and

$$
I_{h}(\phi) \equiv h \sum_{j, n} \phi_{j}^{n}\left(\eta\left(u_{j}^{n+1}\right)-\eta\left(u_{j}^{n}\right)\right)+\frac{1}{2} \lambda \sum_{j, n} \phi_{j}^{n}\left(q\left(u_{j+1}^{n}\right)-q\left(u_{j-1}^{n}\right)\right) .
$$

As (3.37), we have from the Taylor expansion that

$$
\begin{aligned}
I_{h}(\phi)= & h \sum_{j, n} \phi_{j}^{n}\left(\left(\eta_{j}^{\prime}\right)^{\top} F_{j}+\frac{1}{2} \lambda \Delta_{0} q_{j}+\left(\eta_{j}^{\prime}\right)^{\top} H_{j}+\left(\eta_{j}^{\prime}\right)^{\top} J_{j}\right) \\
& +\frac{1}{2} h \sum_{j, n} \phi_{j}^{n}\left(F_{j}+H_{j}+J_{j}\right)^{\top} \eta_{j}^{\prime \prime}\left(F_{j}+H_{j}+J_{j}\right) \\
& +\frac{1}{2} h \sum_{j, n} \phi_{j}^{n} \eta^{\prime \prime \prime}(\xi)\left(F_{j}+H_{j}+J_{j}\right)^{3} \equiv I_{1}^{h}(\phi)+I_{2}^{h}(\phi)+I_{3}^{h}(\phi) .
\end{aligned}
$$


Estimate of $I_{1}^{h}$. The estimate of the last term in $I_{1}^{h}$ is similar to that in (3.38) and (3.39).

$$
\sum_{j, n} \phi_{j}^{n}\left(\eta_{j}^{\prime}\right)^{\top} J_{j} \leq-\frac{1}{2} \lambda \beta_{0} \sum_{j, n}\left(\phi_{j}^{n}+\phi_{j+1}^{n}\right)\left|\Delta_{+} a_{j}\right|^{3}+\lambda C \sum_{j, n}\left|\Delta_{+} u_{j}\right|^{2}\left|\Delta_{+} \phi_{j}^{n}\right| .
$$

Similar to that in (3.41), one can obtain

$$
\begin{aligned}
\sum_{j, n} \phi_{j}^{n}\left(\eta_{j}^{\prime}\right)^{\top} H_{j} \leq & -\frac{1}{2} \lambda^{2} \sum_{j, n} \phi_{j}^{n}\left(\Delta_{+} f_{j}\right)^{\top} \eta_{j}^{\prime \prime} \Delta_{+} f_{j}+\lambda \varepsilon_{0} C \sum_{j, n}\left(\phi_{j}^{n}+\phi_{j+1}^{n}\right)\left|\Delta_{+} u_{j}\right|^{3} \\
& +\lambda \varepsilon_{0} C \sum_{j, n}\left|\Delta_{+} u_{j}\right|^{2}\left|\Delta_{+} \phi_{j}^{n}\right| .
\end{aligned}
$$

The estimate of the first term in $I_{1}^{h}$ is similar to that in (3.40). One has

$$
\begin{aligned}
\sum_{j, n} \phi_{j}^{n}\left(\left(\eta_{j}^{\prime}\right)^{\top} F_{j}+\frac{1}{2} \lambda \Delta_{0} q_{j}\right) & =-\frac{1}{2} \lambda \sum_{j, n} \phi_{j}^{n}\left(\left(\eta_{j}^{\prime}\right)^{\top} \Delta_{0} f_{j}-\Delta_{0} q_{j}\right) \\
& \leq \lambda C \sum_{j, n}\left(\phi_{j}^{n}+\phi_{j+1}^{n}\right)\left|\Delta_{+} u_{j}\right|^{3}+\lambda C \sum_{j, n}\left|\Delta_{+} u_{j}\right|^{2}\left|\Delta_{+} \phi_{j}^{n}\right| .
\end{aligned}
$$

The estimates (5.20)-(5.21) yield

$$
\begin{aligned}
I_{1}^{h}(\phi) \leq & -\frac{1}{2} \lambda \beta_{0} h \sum_{j, n}\left(\phi_{j}^{n}+\phi_{j+1}^{n}\right)\left|\Delta_{+} u_{j}\right|^{3}-\frac{1}{2} \lambda^{2} h \sum_{j, n} \phi_{j}^{n}\left(\Delta_{+} f_{j}\right)^{\top} \eta_{j}^{\prime \prime} \Delta_{+} f_{j} \\
& +\lambda\left(1+\varepsilon_{0}\right) C h \sum_{j, n}\left(\phi_{j}^{n}+\phi_{j+1}^{n}\right)\left|\Delta_{+} u_{j}\right|^{3}+\lambda\left(1+\varepsilon_{0}\right) C h \sum_{j, n}\left|\Delta_{+} u_{j}\right|^{2}\left|\Delta_{+} \phi_{j}^{n}\right| .
\end{aligned}
$$

We can similarly estimate $I_{2}^{h}$ and $I_{3}^{h}$. Finally we have

$$
\begin{aligned}
I_{h}(\phi) \leq & -\frac{1}{2} \lambda \beta_{0} h \sum_{j, n}\left(\phi_{j}^{n}+\phi_{j+1}^{n}\right)\left|\Delta_{+} u_{j}\right|^{3}-\lambda^{2} C h \sum_{j, n} \phi_{j}^{n}\left(\Delta_{-} \Delta_{+} f_{j}\right)^{2} \\
& +\lambda C h \sum_{j, n}\left|\Delta_{+} u_{j}\right|^{2}\left(\left|\Delta_{+} \phi_{j}^{n}\right|+\left|\Delta_{-} \phi_{j}^{n}\right|\right)+\lambda C \varepsilon_{0}^{2} h \sum_{j, n}\left(\phi_{j}^{n}+\phi_{j+1}^{n}\right)\left|\Delta_{+} u_{j}\right|^{3} .
\end{aligned}
$$

We choose $\beta_{0}$ large and $\varepsilon_{0}$ small and then have

$$
I_{h}(\phi) \leq \lambda C h \sum_{j, n}\left|\Delta_{+} u_{j}\right|^{2}\left(\left|\Delta_{+} \phi_{j}^{n}\right|+\left|\Delta_{-} \phi_{j}^{n}\right|\right) \leq h^{\alpha-1 / 3}\|\phi\|_{C^{\alpha}} .
$$

Therefore, we obtain

Proposition 5.2. Under the same assumptions of Proposition 5.1,

$$
\sum_{j, n} \phi_{j}^{n}\left(\eta\left(u_{j}^{n+1}\right)-\eta\left(u_{j}^{n}\right)\right) h+\frac{1}{2} \lambda \sum_{j, n} \phi_{j}^{n}\left(q\left(u_{j+1}^{n}\right)-q\left(u_{j-1}^{n}\right)\right) h \leq C h^{\alpha-1 / 3}\|\phi\|_{C^{\alpha}},
$$

for $C^{2}$ convex entropy pair $(\eta, q)$ and $\phi \in C_{0}^{1}, \phi \geq 0$.

Similar to Section 3.3, we can show that $\partial_{t} \eta\left(u_{h}\right)+\partial_{x} q\left(u_{h}\right)$ is compact in $H_{\text {loc }}^{-1}$ for any convex entropy pair $(\eta, q)$. For a general entropy function we write it as a linear combination of two convex entropy functions as we have explained in Section 4. Hence we have 
Proposition 5.3. Under the same assumptions of Proposition 5.1, $\partial_{t} \eta\left(u_{h}\right)+$ $\partial_{x} q\left(u_{h}\right)$ is compact in $H_{\text {loc }}^{-1}$ for any $C^{2}$ entropy pair $(\eta, q)$.

5.3. Convergence and entropy consistency. Now we are concerned with the convergence of uniformly bounded approximate solutions of $2 \times 2$ systems of conservation laws endowed with global Riemann invariants and the entropy consistency of boundedly convergent Lax-Wendroff numerical solutions of general hyperbolic systems of conservation laws with a convex entropy.

One important example of the $2 \times 2$ systems is the elasticity equations (cf. [5]):

$$
\partial_{t} v-\partial_{x} u=0, \quad \partial_{t} u-\partial_{x} \sigma(v)=0,
$$

where $u$ is the specific volume, $v$ is the strain, and $\sigma$ is the stress-strain relation satisfying $\sigma^{\prime}(v)>0$. The numerical experiments indicate that the Lax-Wendroff approximate solutions $\left(v_{h}, u_{h}\right)$ are uniformly bounded. It is important from the viewpoint of numerical analysis whether uniformly bounded Lax-Wendroff numerical solutions may generate some oscillations. The following theorem shows that, if $\left(v_{h}, u_{h}\right)$ is uniformly bounded, then it indeed strongly converges to the corresponding entropy solution. The proof follows from Theorem 2.2, the estimates in Sections 5.1 and 5.2, and similar compactness arguments as in the scalar cases.

Theorem 5.1. Consider a $2 \times 2$ genuinely nonlinear and strictly hyperbolic system of conservation laws (1.1) endowed with global Riemann invariants. Let $u_{h}(x, t)$ be the Lax-Wendroff approximate solutions. Assume that $u_{h}$ is uniformly bounded and the coefficient $\beta$ in (5.2) is suitably large and the CFL number $\varepsilon_{0}$ given by (1.3) is suitably small. Then there is a subsequence (still denoted as) $u_{h}$ such that $u_{h}(x, t) \rightarrow u(x, t) \quad$ a.e. as $h \rightarrow 0$ and $u$ is the entropy solution of (1.1) satisfying $\partial_{t} \eta(u)+\partial_{x} q(u) \leq 0 \quad$ in $\mathcal{D}^{\prime}$ for any convex entropy $(\eta, q)$.

For a general system, we conclude that the numerical solutions of the LaxWendroff scheme, if they are boundedly convergent, then converge to the entropy solutions. A similar result has been obtained by Majda and Osher [15] for the semi-discrete case of time-dependent systems and for the fully discrete case of timeindependent systems.

Theorem 5.2. Consider a general system of genuinely nonlinear and strictly hyperbolic conservation laws (1.1) endowed with a convex entropy. Let $u_{h}(x, t)$ be the uniformly bounded Lax-Wendroff approximate solutions converging boundedly a.e. to $u(x, t)$. Assume that the coefficient $\beta$ in (5.2) is suitably large and the CFL number $\varepsilon_{0}$ given by (1.3) is suitably small. Then $u$ is the entropy solution of (1.1) satisfying $\partial_{t} \eta(u)+\partial_{x} q(u) \leq 0$ in $\mathcal{D}^{\prime}$ for any convex entropy pair $(\eta, q)$.

A typical example for such a system is the system of gas dynamics in Lagrangian coordinates:

$$
\partial_{t}\left(\begin{array}{c}
v \\
u \\
E
\end{array}\right)+\partial_{x}\left(\begin{array}{c}
-u \\
p \\
p u
\end{array}\right)=0,
$$

where $v>0$ denotes the specific volume of the gas, $u$ the velocity, $p>0$ the pressure, $E=\frac{1}{2} u^{2}+e$ the total specific energy and $e$ the specific internal energy. The additional conservation law assumes the form $\partial_{t}(-S) \leq 0$ for physical solutions. For polytropic gases, $-S(v, u, E)=-\log \left(E-\frac{1}{2} u^{2}\right)-(\gamma-1) \log v$ is a strictly convex function of the state variables $v, u$, and $E$ for $\gamma>1$, a fixed constant, 
and $p=(\gamma-1) \frac{E-\frac{1}{2} u^{2}}{v}=A(S) v^{\gamma}$. Theorem 5.2 indicates that uniformly bounded convergent Lax-Wendroff approximation is consistent with this system.

\section{The MacCormack scheme and Richtmyer scheme}

An efficient implementation of the Lax-Wendroff scheme is to use two step splitting methods. One particularly popular version is the following MacCormack scheme:

$$
\begin{aligned}
\tilde{u}_{j}^{n} & =u_{j}^{n}-\lambda \Delta_{+} f\left(u_{j}^{n}\right), \\
u_{j}^{n+1} & =\frac{1}{2}\left(u_{j}^{n}+\tilde{u}_{j}^{n}\right)-\lambda \Delta_{-} f\left(\tilde{u}_{j}^{n}\right)+\lambda_{-}\left(\beta_{j+1 / 2}^{n}\left|\Delta_{+} f^{\prime}\left(u_{j}^{n}\right)\right| \Delta_{+} u_{j}^{n}\right) .
\end{aligned}
$$

The convergence of this scheme is very much similar to what we did for the LaxWendroff scheme in Sections 3-5. We only state some convergence theorems and show those arguments that are different from ones of the previous sections.

We first rewrite the MacCormack scheme (6.1) as follows

$$
u_{j}^{n+1}=u_{j}^{n}+F_{j}^{n}+H_{j}^{n}+\tilde{H}_{j}^{n}+J_{j}^{n},
$$

where

$$
\begin{aligned}
& F_{j}^{n}=-\frac{1}{2} \lambda_{n} \Delta_{0} f\left(u_{j}^{n}\right), \\
& H_{j}^{n}=\frac{1}{2} \lambda_{n}^{2} \Delta_{-}\left(\frac{\left(\Delta_{+} f\left(u_{j}^{n}\right)\right)^{2}}{\Delta_{+} u_{j}^{n}}\right), \\
& \tilde{H}_{j}^{n}=\frac{1}{2} \lambda_{n} \Delta_{-}\left(f\left(u_{j}^{n}\right)-f\left(\tilde{u}_{j}^{n}\right)-f^{\prime}\left(u_{j}^{n}\right)\left(u_{j}^{n}-\tilde{u}_{j}^{n}\right)\right), \\
& J_{j}^{n}=\lambda_{n} \Delta_{-}\left(\tilde{\beta}_{j+1 / 2}^{n}\left|\Delta_{+} a\left(u_{j}^{n}\right)\right| \Delta_{+} u_{j}^{n}\right), \\
& \tilde{\beta}_{j+1 / 2}^{n}=\beta_{j+1 / 2}^{n}+\lambda_{n} \frac{a\left(u_{j}^{n}\right)-\Delta_{+} f_{j} / \Delta_{+} u_{j}}{\left|\Delta_{+} a_{j}\right|} .
\end{aligned}
$$

Clearly, we can carry out the analysis without any difference in the absence of the term $\tilde{H}_{j}^{n}$. We only point out that $\tilde{H}_{j}^{n}$ can only produce third-order terms and can be controlled by the viscosity term based on the following facts.

First, we notice that $\tilde{u}^{n}$ in (6.1) is always in the interval $\left[u_{j}^{n}, u_{j+1}^{n}\right]$ provided the CFL number is less than $1 / 2$. Using the Taylor expansion and (6.1), one has

$$
f\left(u_{j}^{n}\right)-f\left(\tilde{u}_{j}^{n}\right)-f^{\prime}\left(u_{j}^{n}\right)\left(u_{j}^{n}-\tilde{u}_{j}^{n}\right)=\frac{1}{2} f^{\prime \prime}\left(\xi_{j}^{n}\right)\left(u_{j}^{n}-\tilde{u}_{j}^{n}\right)^{2}=\frac{1}{2} \lambda^{2} f^{\prime \prime}\left(\xi_{j}^{n}\right)\left(\Delta_{+} f\left(u_{j}^{n}\right)\right)^{4} .
$$

Substituting (6.4) into $\tilde{H}_{j}$ and using the summation by parts, one has

$$
\begin{gathered}
\sum_{j} \eta^{\prime}\left(u_{j}^{n}\right) \tilde{H}_{j}^{n}=-\frac{1}{4} \lambda^{3} \sum_{j} \Delta_{+} \eta_{j}^{\prime} f^{\prime \prime}\left(\xi_{j}^{n}\right)\left(\Delta_{+} f\left(u_{j}^{n}\right)\right)^{4} \leq \lambda \varepsilon_{0}^{2} C \sum_{j} \Phi\left(s_{j}^{n}\right)\left|\Delta_{+} u_{j}^{n}\right|^{3}, \\
\sum_{j} \eta^{\prime \prime}\left(u_{j}^{n}\right)\left(\tilde{H}_{j}^{n}\right)^{2}=\frac{1}{4} \lambda^{6} \sum_{j} \eta^{\prime \prime}\left(u_{j}^{n}\right)\left(\Delta_{-}\left(a^{\prime}\left(\xi_{j}^{n}\right)\left(\Delta_{+} f\left(u_{j}^{n}\right)\right)^{2}\right)\right)^{2} \\
\leq \lambda \varepsilon_{0}^{5} C \sum_{j} \Phi\left(s_{j}^{n}\right)\left|\Delta_{+} u_{j}^{n}\right|^{3},
\end{gathered}
$$


and

$$
\begin{aligned}
\sum_{j} \eta^{\prime \prime}\left(u_{j}^{n}\right) F_{j}^{n} \tilde{H}_{j}^{n} & =-\frac{1}{8} \lambda^{4} \sum_{j} \eta^{\prime \prime}\left(u_{j}^{n}\right) \Delta_{0} f\left(u_{j}^{n}\right) \Delta_{-}\left(a^{\prime}\left(\xi_{j}^{n}\right)\left(\Delta_{+} f\left(u_{j}^{n}\right)\right)^{2}\right) \\
& \leq \lambda \varepsilon_{0}^{3} C \sum_{j} \Phi\left(s_{j}^{n}\right)\left|\Delta_{+} u_{j}^{n}\right|^{3} .
\end{aligned}
$$

Now we treat another two step version of the Lax-Wendroff scheme - the Richtmyer scheme,

$$
\begin{aligned}
\tilde{u}_{j+1 / 2}^{n} & =\frac{1}{2}\left(u_{j}^{n}+u_{j+1}^{n}\right)-\frac{1}{2} \lambda \Delta_{+} f\left(u_{j}^{n}\right), \\
u_{j}^{n+1} & =u_{j}^{n}-\lambda \Delta_{-} f\left(\tilde{u}_{j+1 / 2}^{n}\right)+\lambda \Delta_{-}\left(\beta_{j+1 / 2}^{n}\left|\Delta_{+} f^{\prime}\left(u_{j}^{n}\right)\right| \Delta_{+} u_{j}^{n}\right) .
\end{aligned}
$$

We can rewrite it into the form

$$
u_{j}^{n+1}=u_{j}^{n}+F_{j}^{n}+H_{j}^{n}+\tilde{H}_{j}^{n}+J_{j}^{n},
$$

where

$$
\begin{aligned}
F_{j}^{n} & =-\frac{1}{2} \lambda_{n} \Delta_{0} f\left(u_{j}^{n}\right), \\
H_{j}^{n} & =\frac{1}{2} \lambda_{n}^{2} \Delta_{-}\left(\frac{\left(\Delta_{+} f_{j}^{n}\right)^{2}}{\Delta_{+} u_{j}^{n}}\right), \\
\tilde{H}_{j}^{n} & =\frac{1}{2} \lambda_{n} \Delta_{-}\left(f\left(u_{j}^{n}\right)+f\left(u_{j+1}^{n}\right)-2 f\left(\tilde{u}_{j+1 / 2}^{n}\right)-f^{\prime}\left(u_{j+1 / 2}^{n}\right)\left(u_{j}^{n}+u_{j+1}^{2}-2 \tilde{u}_{j}^{n}\right)\right), \\
J_{j}^{n} & =\lambda_{n} \Delta_{-}\left(\tilde{\beta}_{j+1 / 2}^{n}\left|\Delta_{+} a\left(u_{j}^{n}\right)\right| \Delta_{+} u_{j}^{n}\right), \quad \tilde{\beta}_{j+1 / 2}^{n}+\lambda_{n} \frac{a_{j}^{n}-\Delta_{+} f_{j}^{n} / \Delta_{+} u_{j}^{n}}{\left|\Delta_{+} u_{j}^{n}\right|} .
\end{aligned}
$$

It is also easy to verify that $\tilde{H}_{j}^{n}$ can produce only third-order terms and can be controlled by the viscosity term.

Therefore we have the following theorems corresponding to those of the LaxWendroff scheme in Sections 3-5 under the same assumptions.

Theorem 6.1. Let $u_{h}(x, t)$ be approximate solutions of the convex scalar conservation laws (1.1) and (3.1) by the the MacCormack scheme (6.1) or the Richtmyer scheme (6.8). Then there is a subsequence strongly converging to the weak solution of (1.1) satisfying the entropy inequalities.

Theorem 6.2. Let $u_{h}(x, t)$ be approximate solutions of the scalar conservation laws (1.1) or (4.4) by the MacCormack scheme (6.1) or by the Richtmyer scheme (6.8) with the artificial viscosity term (4.1). Then there is a subsequence strongly converging to the weak solution of (1.1) satisfying the entropy inequalities.

Theorem 6.3. Consider a general $2 \times 2$ genuinely nonlinear and strictly hyperbolic system of conservation laws (1.1) endowed with global Riemann invariants. Let $u_{h}(x, t)$ be the uniformly bounded approximate solutions of the MacCormack scheme (6.1) or the Richtmyer scheme (6.8). Then there is a subsequence (still denoted as $) u_{h}$ such that $u_{h}(x, t) \rightarrow u(x, t)$ a.e. as $h \rightarrow 0$ and $u$ is the entropy solution of (1.1) satisfying $\partial_{t} \eta(u)+\partial_{x} q(u) \leq 0$ in $\mathcal{D}^{\prime}$ for any $C^{2}$ convex entropy pair $(\eta, q)$.

Theorem 6.4. Consider a general system of hyperbolic conservation laws (1.1) endowed with a convex entropy. Let $u_{h}(x, t)$ be the approximate solutions of the MacCormack scheme (6.1) or the Richtmyer scheme (6.8). If $u_{h}$ converges boundedly 
a.e. $u(x, t)$, then $u(x, t)$ is the entropy solution of $(1.1)$ satisfying $\partial_{t} \eta(u)+\partial_{x} q(u) \leq$ 0 in $\mathcal{D}^{\prime}$ for any $C^{2}$ convex entropy pair $(\eta, q)$.

\section{ACKNOWLEDGMENTS}

The authors would like to thank Professor P. D. Lax for his encouragements and Professors J. Goodman, T.-P. Liu, A. Majda, S. Osher, and E. Tadmor for their interest in this work. G.-Q. Chen was supported in part by ONR grant N0001491-J-1384, NSF grant DMS-9623203, and Alfred P. Sloan Foundation Fellowship. J.-G. Liu was supported in part by NSF Grant DMS-9114456, DOE grant DEFG02-88ER25053 and ARO Grant DAAL03-92-G-0143. This work was initiated while the authors visited Stanford University and was supported in part through Army grant DAAL 03-91-G-0017.

\section{REFERENCES}

1. J.P. Boris and D.L. Book, Flux corrected transport. I. SHASTA, a fluid transport algorithm that works, J. Comp. Phys. 11 (1973), 38-69.

2. G.-Q. Chen, The compensated compactness method and the system of isentropic gas dynamics, MSRI Preprint 00527-91, Berkeley (1990).

3. G.-Q. Chen and Y.-G. Lu, A study on application approaches of the theory of compensated compactness, Chinese Science Bulletin 34 (1989), 15-19. MR 90h:35024

4. F. Coquel and P. Le Floch, Convergence of finite difference schemes for conservation laws in several space variables: the corrected antidiffusive flux approach, Math. Comp. 57 (1991), 169-210. MR 91m:65229

5. R. DiPerna, Convergence of approximate solutions to conservation laws, Arch. Rat. Mech. Anal. 82 (1983), 27-70. MR 84k:35091

6. A. Harten, B. Engquist, S. Osher, and S. Chakravarthy, Uniformly high order accurate nonoscillatory schemes, III, J. Comp. Phys. 71 (1987), 231-303. MR 90a:65199

7. A. Harten, J.M. Hyman, and P.D. Lax, On finite-difference approximations and entropy conditions for shocks, Comm. Pure Appl. Math. 29 (1976), 297-322. MR 54:1640

8. A. Harten, P. D. Lax, and B. van Leer, On upstream differencing and Godunov-type schemes for hyperbolic conservation laws, SIAM Review 25 (1983), 35. MR 85h:65188

9. S. Jin and Z. Xin, The relaxing schemes for systems of conservation laws in arbitrary space dimensions, Preprint (1993).

10. P.D. Lax and C.D. Levermore and S. Venakides, The generation and propagation of oscillations in dispersive IVPs and their limiting behavior, Important developments in soliton theory 1980-1990, T. Fokas and V.E. Zakharov eds, Springer-Verlag, Berlin, 1992. MR 95c:35245

11. P.D. Lax and B. Wendroff, Systems of conservation laws, Comm. Pure Appl. Math. 13 (1960), 217-237. MR 22:11523

12. P.L. Lions and P. Souganidis, Convergence of MUSCL type methods for scalar conservation laws, C. R. Acad. Sci. Paris, Série I 311 (1990), 259-264. MR 91i:65168

13. R.W. MacCormack, The effect of viscosity in hyperbolicity impact cratering, AIAA Paper (1969), 69-354.

14. A. Majda and S. Osher, A systematic approach for correcting nonlinear instability: the Lax-Wendroff scheme for scalar conservation laws, Num. Math. 30 (1978), 429-452. MR 80g:65101

15. A. Majda and S. Osher, Numerical viscosity and the entropy condition, Comm. Pure Appl. Math. 32 (1979), 797-838. MR 80j:65031

16. C.S. Morawetz, An alternative proof of DiPerna's theorem, Comm. Pure Appl. Math. 44 (1991), 1081-1090. MR 92m:35165

17. F. Murat, L'injection du cone positif de $H^{-1}$ dans $W^{-1, q}$ est compacte pour tout $q<2$, J. Math. Pures Appl. 60 (1981), 309-322. MR 83b:46045

18. H. Nessyahu and E. Tadmor, Non-oscillatory central differencing for hyperbolic conservation laws, J. Comp. Phys. 87 (1990), 408-463. MR 91i:65157

19. S. Osher, On convergence of generalized MUSCL schemes, SIAM J. Numer. Anal. 22 (1985), 947-961. MR 87b:65147 
20. S. Osher and E. Tadmor, On the convergence of difference approximations to scalar conservation laws, Math. Comp. 50 (1988), 19-51. MR 89m:65086

21. B. Perthame, Second-order Boltzmann schemes for compressible Euler equations in one and two space dimensions, SIAM J. Numer. Anal. 29 (1992), 1-19. MR 92m:76111

22. R.D. Richtmyer and K.W. Morton, Difference Methods for Initial Value Problems, 2nd ed., Wiley-Interscience, New York, 1967. MR 36:3515

23. D. Serre, La compacité par compensation pour les systèmes hyperboliques non linéaires de deux équations à une dimension d'espace, J. Math. Pures Appl. 65 (1986), 423-468. MR 88d:35123

24. C.-W. Shu and S. Osher, Efficient implementation of essentially non-oscillatory shock capturing schemes, J. Comp. Phys. 83 (1989), 32-51. MR 90i:65167

25. Y.S. Smyrlis, Existence and stability of stationary profiles of the LW scheme, Comm. Pure Appl. Math. 43 (1990), 509-545. MR 91d:65143

26. P.R. Sweby, High resolution schemes using flux limiters for hyperbolic conservation laws, SIAM J Num. Anal. 21 (1984), 995-1011. MR 85m:65085

27. T. Tang, On three-point second-order accurate conservative difference schemes, J. Comp. Math. 5 (1987), 105-118. MR 89g:65115

28. L. Tartar, Compensated compactness and applications to partial differential equations, Research Notes in Mathematics, Nonlinear Analysis and Mechanics, ed. R.J. Knops, vol. 4, Pitman Press, New York, 1979. MR 81m:35014

29. B. van Leer, Towards the ultimate conservative difference schemes, $V, A$ second order sequel to Godunov's method, J. Comp. Phys. 43 (1981), 357-372.

30. P. Woodward and P. Colella, The numerical simulation of two dimensional fluid flow with strong shock, J. Comp. Phys. 54 (1984), 115-173. MR 85e:76004

31. H. Yang, Nonlinear wave analysis and convergence of MUSCL schemes, IMA Preprint 697 (1990).

32. S.-H. Yu, Existence of the local discrete shock profile for the Lax-Wendroff scheme, Preprint (1992).

Department of Mathematics, Northwestern University, Evanston, Illinois 60208

E-mail address: gqchen@math.nwu.edu

Department of Mathematics, Temple University, Philadelphia, Pennsylvania 19122

E-mail address: jliu@math.temple.edu 